FERMILAB-TM-2055

\title{
Impedances and Collective Instabilities of the Tevatron at Run II
}

\author{
King-Yuen Ng \\ Fermi National Accelerator Laboratory \\ P.O. Box 500, Batavia, Illinois 60510
}

September 1998 


\section{Disclaimer}

This report was prepared as an account of work sponsored by an agency of the United States Government. Neither the United States Government nor any agency thereof, nor any of their employees, makes any warranty, expressed or implied, or assumes any legal liability or responsibility for the accuracy, completeness, or usefulness of any information, apparatus, product, or process disclosed, or represents that its use would not infringe privately owned rights. Reference herein to any specific commercial product, process, or service by trade name, trademark, manufacturer, or otherwise, does not necessarily constitute or imply its endorsement, recommendation, or favoring by the United States Government or any agency thereof. The views and opinions of authors expressed herein do not necessarily state or reflect those of the United States Government or any agency thereof.

\section{Distribution}

Approved for public release; further dissemination unlimited. 
TM-2055

\title{
IMPEDANCES AND COLLECTIVE INSTABILITIES OF THE TEVATRON AT RUN II
}

\author{
King-Yuen Ng \\ Fermi National Accelerator Laboratory,* P.O. Box 500, Batavia, IL 60510
}

(May 1998)

\begin{abstract}
The longitudinal and transverse coupling impedances of the Tevatron vacuum chamber are estimated and summed up. The resistive-wall impedances of the beam pipe and the laminations in the Lambertson magnets dominate below $\sim 50 \mathrm{MHz}$. Then come the inductive parts of the bellows and BPM's. The longitudinal and transverse collective instabilities, for both single bunch and multi bunches, are studied using Run II parameters. As expected the transverse coupled-bunch instability driven by the resistivewall impedance is the most severe collective instability. However, it can be damped by a transverse damper designed for the correction of injection offsets. The power of such a damper has been studied.
\end{abstract}

*Operated by the Universities Research Association, Inc., under contract with the U.S. Department of Energy. 


\section{CONTENTS}

I Introduction

II Coupling impedances

II.1 Resistive wall

II.2 Lambertson magnets

II.3 Beam-position monitors

II.4 Bellows

II.5 Separators

II.6 RF cavities

II.7 Summary

III Potential-well distortion

IV Longitudinal microwave instability

V Longitudinal coupled-bunch instabilities

VI Longitudinal head-tail instability

VII Transverse microwave instability

VIII Transverse coupled-bunch instabilities

VIII.1 Resistive wall

VIII.2 Sinusoidal modes

VIII.3 Transverse coupled-bunch instability driven by resonances

XI Transverse head-tail instability

$\mathrm{X}$ Fast transverse damper

XI Conclusions

Acknowledgment

References 


\section{INTRODUCTION}

Tevatron Run II will be very different from Run I because there will be more proton and antiproton bunches. Also the intensities of the bunches are different, and the Run II bunch lengths are shorter. Here, we are going to give a detailed analysis of the collective instabilities to be encountered in this new run. The run parameters are listed in Table I.

First, in Sec. II, the longitudinal and transverse coupling impedances of the Tevatron vacuum chamber will be studied. These include the resistive wall, the Lambertson magnets, the beam-position monitors, the bellows, the separators, and the rf cavities. In Sec. III, the effect of potential-well distortion driven by the inductive part of the impedance is reviewed. Section IV deals with the longitudinal microwave instability and Sec. V with the longitudinal coupled-bunch instability. Sec. VI is devoted to the longitudinal head-tail instability which had been observed during Run I. Transverse microwave instability is pursued in Sec. VI. We follow Sacherer's analysis of transverse coupled-bunch instabilities driven by both the resistive wall and sharp resonances of the rf cavities in Sec. VIII. The issue of transverse head-tail instabilities resulting from a finite chromaticity is dealt with in Sec. IX. We discuss briefly, in Sec. X, the power required by the transverse damper to correct an injection error and to cure the transverse dipole-mode collective instabilities. Finally, in Sec. XI, we give a conclusion about the coupling impedances, the most dangerous collective instabilities, and possible cures.

\section{COUPLING IMPEDANCES}

\section{II.1 RESISTIVE WALL}

The Tevatron beam pipe is square in cross section with sides $h=6.0 \mathrm{~cm}$ and rounded corners. The longitudinal and transverse impedances of the Tevatron due to wall resistivity at frequency $\omega /(2 \pi)$ are $[1]$

$$
\begin{gathered}
Z_{\|}=[1+j \operatorname{sgn}(\omega)] \frac{\rho C}{\pi \delta h}, \\
Z_{\perp}=[1+j \operatorname{sgn}(\omega)] \frac{8 c \rho C}{\pi \omega \delta h^{3}},
\end{gathered}
$$

where $\rho=7.4 \times 10^{-7} \Omega$-m is the resistivity of the stainless steel wall and

$$
\delta=\sqrt{\frac{2 \rho}{|\omega| \mu_{0} \mu_{r}}}=\sqrt{\frac{2 \rho c}{|\omega| Z_{0}}},
$$

is the skin depth. In the above, $\mu_{0}$ and $Z_{0} \approx 377 \Omega$ are, respectively, the magnetic permeability and impedance of free space, and the relative magnetic permeability of the beam-pipe wall has been taken as $\mu_{r} \approx 1$. Note that we have been writing the formulas for impedances in such a way that they are valid for both positive and negative frequencies. This is important, especially because it is the real parts of $Z_{\|}$and $Z_{\perp}$ at negative frequencies that drive almost all the collective instabilities.

Putting in the ring circumference $C=2 \pi R$ with $R=1 \mathrm{~km}$, we obtain

$$
\frac{Z_{\|}}{n}=[\operatorname{sgn}(\omega)+j] 12.45|n|^{-1 / 2} \Omega
$$


Table I: Performance parameters for Run II.

\begin{tabular}{|c|c|c|c|}
\hline Mode $\left(n_{p} \times n_{\bar{p}}\right)$ & $36 \times 36$ & $140 \times 121$ & \\
\hline$p$ per bunch $N_{p}$ & $2.70 \times 10^{11}$ & $2.70 \times 10^{11}$ & \\
\hline $\bar{p}$ per bunch $N_{\bar{p}}$ & $3.00 \times 10^{10}$ & $3.00 \times 10^{10}$ & \\
\hline Total $\bar{p}$ & $1.08 \times 10^{12}$ & $3.63 \times 10^{12}$ & \\
\hline Proton emittance $\epsilon_{p}$ & 20 & 20 & mm-mrad \\
\hline Antiproton emittance $\epsilon_{\bar{p}}$ & 15 & 15 & mm-mrad \\
\hline Number of $\bar{p}$ bunches & 36 & 121 & \\
\hline Low beta $\beta^{*}$ & 35 & 35 & $\mathrm{~cm}$ \\
\hline Crossing angle & 0 & 136 & $\mu \mathrm{rad}$ \\
\hline Bunch spacing & 395.44 & 131.81 & ns \\
\hline Luminosity & $8.60 \times 10^{31}$ & $1.61 \times 10^{32}$ & $\mathrm{~cm}^{-2} \mathrm{~s}^{-1}$ \\
\hline Events per crossing & 2.3 & 1.3 & \\
\hline Total energy $E$ & 1000 & 1000 & $\mathrm{GeV}$ \\
\hline Rms bunch length & 0.370 & 0.370 & $\mathrm{~m}$ \\
\hline or & 1.234 & 1.234 & ns \\
\hline Rf voltage $V_{\mathrm{rf}}$ & 1.00 & 1.00 & MV \\
\hline Rf harmonic $h$ & 1113 & 1113 & \\
\hline Slippage factor $\eta$ & 0.002827 & 0.002827 & \\
\hline Synchrotron tune $\nu_{s}$ & $7.077 \times 10^{-4}$ & $7.077 \times 10^{-4}$ & \\
\hline Rms momentum spread & $9.262 \times 10^{-5}$ & $9.262 \times 10^{-5}$ & \\
\hline Rms bunch area & 0.3591 & 0.3591 & $\mathrm{eV}-\mathrm{s}$ \\
\hline
\end{tabular}

where $\nu_{\beta}$ is the betatron tune.

For high frequencies, a more accurate expression for the resistive-wall impedances is [2]

$$
Z_{m}^{\|}=\frac{\omega}{c} Z_{m}^{\perp}=\frac{Z_{0} C c}{\pi}\left(\frac{2}{h}\right)^{2 m}\left\{[1-\operatorname{sgn}(\omega) j]\left(1+\delta_{m 0}\right) \frac{h c}{2} \sqrt{\frac{Z_{0} c}{2 \rho|\omega|}}-\frac{j h^{2} \omega}{4(m+1)}-\frac{j m c^{2}}{\omega}\right\}^{-1} .
$$

What we have discussed so for are the lowest azimuthals $m$; therefore the longitudinal impedance $Z_{\|}$corresponds to $Z_{0}^{\|}$in Eq. (2.6) and $Z_{\perp}$ corresponds to $Z_{1}^{\perp}$. We see that the resistive-wall impedances will follow Eqs. (2.4) and (2.5) for all practical frequencies, because they will roll off only at very high frequencies when

$$
f \gtrsim \frac{c}{2 \pi}\left(\frac{2 Z_{0}}{\rho h^{2}}\right)^{1 / 3}=313 \mathrm{GHz} .
$$

\section{II.2 LAMBERTSON MAGNETS}

The main concern of the Lambertson magnets is the low-frequency component created by the exposure 
of the beam to the bare laminations of the magnets. A rough estimation of the Lambertson magnets is made by approximating the magnet as a series of annular laminations of $0.953 \mathrm{~mm}$ thick separated by cracks of width $\Delta$ which is $3 \%$ of the lamination thickness. The inner radius is chosen to be $b=3.0 \mathrm{~cm}$ and the outer radius $d=8.0 \mathrm{~cm}$. The low-frequency image current traveling through the magnet is assumed to flow in one lamination from the inner radius to the outer radius then cross over to the next lamination and flow from the outer radius to the inner radius. Even though we are concerned about the low-frequency impedance, due to the high relative magnetic permeability of the lamination, the skin depth for the frequencies we are considering is still less than the lamination thickness so that the current is constrained to one skin depth in the laminations. In this way the total resistance of the magnet is found by adding up the resistance along the entire current path.

For the current traveling from the inner radius to the outer radius the net impedance is found to be

$$
Z_{\|}=[1+j \operatorname{sgn}(\omega)] \frac{\rho_{\ell}}{\pi \delta_{\ell}} \ln \frac{d}{b},
$$

where $\rho_{\ell}$ is the resistivity of the laminations and $\delta_{\ell}$ is the skin depth. For the current traveling along the inner tip of the laminations the resistance per unit length is

$$
Z_{\|}=[1+j \operatorname{sgn}(\omega)] \frac{\rho_{\ell}}{2 \pi b \delta_{\ell}} .
$$

There are four 110.25-inch Lambertson magnets, or $11.20 \mathrm{~m}$ in total. We use a resistivity of $\rho_{\ell} \approx$ $2 \times 10^{-7} \Omega$-m and a relative permeability of $\mu_{r} \approx 100$ for the lamination material. The total low frequency resistive wall impedance around the laminations is calculated to be

$$
Z_{\|} / n=[\operatorname{sgn}(\omega)+j] \frac{7.237}{\sqrt{|n|}} \Omega .
$$

To estimate the transverse impedance we use the approximate relation

$$
Z_{\perp}=\frac{2 c}{b^{2}} \frac{Z_{\|}}{\omega}
$$

and arrive at

$$
Z_{\perp}=[\operatorname{sgn}(\omega)+j] 16.08\left|n+\nu_{\beta}\right|^{-1 / 2} \mathrm{M} \Omega / \mathrm{m} .
$$

It should be noted that the Lambertson magnets were assumed to have an circular geometry with inner radius of $b=3.0 \mathrm{~cm}$. The actual shape of the Lambertson is much different so this estimate can only be approximate. Using a slightly larger inner radius can change the impedance by a significant amount; for example, if $b$ is $10 \%$ larger the transverse impedance will drop by $\sim 25 \%$.

Therefore, at low frequencies, the total impedances due to the stainless steel beam pipe and the Lambertson magnets add up to

$$
\begin{gathered}
\frac{Z_{\|}}{n}=[\operatorname{sgn}(\omega)+j] 19.680|n|^{-1 / 2} \Omega, \\
Z_{\perp}=[\operatorname{sgn}(\omega)+j] 43.74\left|n+\nu_{\beta}\right|^{-1 / 2} \mathrm{M} \Omega / \mathrm{m} .
\end{gathered}
$$

At higher frequencies, the cracks between the laminations of the Lambertsons behave like radial transmission lines. We assume that the medium in the cracks of width $\Delta \approx 28.6 \mu \mathrm{m}$ has a dielectric constant 
$\epsilon_{c} \approx 6$, a relative magnetic permeability of $\mu_{c} \approx 1$, and a high resistivity of $\rho_{c} \approx 100 \Omega$-m. At radius $r$ inside the crack, the series impedance per unit radial length is

$$
Z=\frac{j \omega Z_{0} \mu_{c}}{c} \frac{\Delta}{2 \pi r}+[1+\operatorname{sgn}(\omega) j] \frac{2 \rho_{\ell}}{2 \pi r \delta_{\ell}},
$$

where the first term is the inductive contribution of the crack medium and the second term the resistivity of the lamination walls depicted in Eq. (2.8). The shunt admittance per unit length is

$$
Y=\left(\frac{j \omega \epsilon_{c}}{Z_{0} c}+\frac{1}{\rho_{m}}\right) \frac{2 \pi r}{\Delta},
$$

which represents the capacitance and shunt resistance of the crack. The wave number of the transmission line is

$$
\beta_{c}=\sqrt{-Z Y}
$$

which is $r$ independent. The characteristic impedance is

$$
Z_{c}=\sqrt{\frac{Z}{Y}},
$$

which is a monotonic decreasing function of frequency. The longitudinal impedance seen by the beam is therefore

$$
Z_{\|}=Z_{c} \tan \beta_{c} d_{c}
$$

where $d_{c}=d-b=5 \mathrm{~cm}$ is the depth of the crack or transmission line. Note that Eq. (2.19) reproduces the low-frequency impedance of Eq.(2.8).

To study the resonances, first let us neglect the resistivity of the crack medium and also the lamination walls. Then the wave number in Eq. (2.17) simplifies to $\beta_{c}=\sqrt{\epsilon_{c} \mu_{c}} \omega / c$. From Eq. (2.19), the $n$th resonance occurs at the frequency

$$
f_{n}=(2 n-1) \frac{c}{4 d_{c} \sqrt{\epsilon_{c} \mu_{c}}},
$$

or $0.612,1.835,3.060, \cdots \mathrm{GHz}$ for the first few. From Eq. (2.15), it is evident that the addition of the wall inductance is equivalent to replacing the permeability of the crack medium by

$$
\mu_{c} \longrightarrow \mu_{c}\left(1+\frac{1}{\mu_{c} \Delta} \sqrt{\frac{\rho_{\ell} \mu_{\ell} c}{Z_{0}|\omega|}}\right)
$$

which is now frequency dependent. Substituting into Eq. (2.20), we find that the wall inductance reduces the resonance frequencies to $0.250,0.979,1.813, \cdots \mathrm{GHz}$. When the real part of the wall resistivity is included, these resonances are highly damped and the resonant frequencies further reduced. The numerical computations of the longitudinal and transverses impedances for the Lambertson magnets are plotted in Fig. 1 up to $1 \mathrm{GHz}$. The transverse impedance $Z_{\perp}$ is estimated from the longitudinal $Z_{\|} / n$ using the relation (2.11). Therefore, they just differ by a constant and are plotted as the same curves but different scales in the figure. Notice that the resonances are so much damped that only the first one survives and has its frequency shifted to $\sim 0.195 \mathrm{GHz}$. The small conductivity of the cracks plays a negligible role because it is very much less than the lamination conductivity. It is worthy to point out that the higher-order resonances do not show up because both $Z_{\|} / n$ and the characteristic impedance $Z_{c}$ decrease with frequency. We also see from Fig. 1 that the impedances $Z_{\|} / n$ and $Z_{\perp}$ have the $n^{-1 / 2}$ low-frequency behavior of Eqs. (2.10) and 


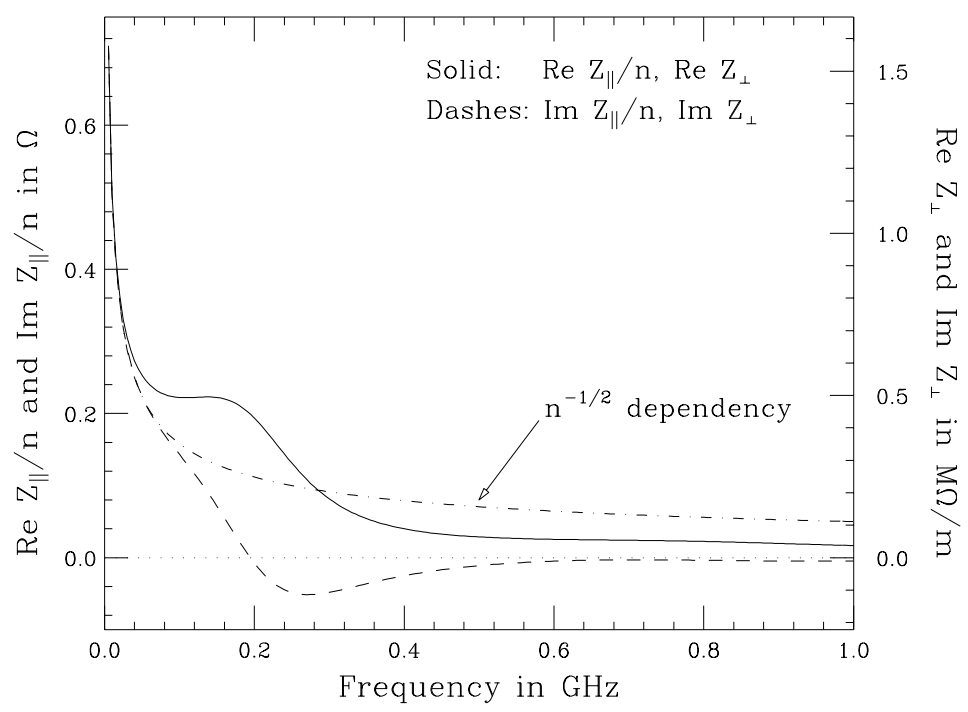

Figure 1: The real and imaginary parts of $Z_{\|} / n$ and $Z_{\perp}$ as functions of frequency for the Tevatron Lambertson magnets. Note that $Z_{\|} / n$ and $Z_{\perp}$ are drawn as the same curves but at different scales.

(2.12), which are also plotted in the figure as reference. They start to deviate from this behavior only near the first damped resonance. Actually, apart from this damped resonance, the impedances do not deviate too much from the $n^{-1 / 2}$ behavior even at higher frequencies.

\section{II.3 BEAM-POSITION MONITORS}

There are $M=216$ sets of beam position monitors (BPM's) in the Tevatron; half of them detect horizontally and half vertically. Each BPM consists of 2 cylindrical strip-lines of radius $b=3.5 \mathrm{~cm}$, each subtending an angle $\phi_{0}=110^{\circ}$ at the center of the beam pipe and is of length $\ell=18 \mathrm{~cm}$. Each strip-line is terminated at both ends and forms a transmission line of characteristic impedance $Z_{c}=50 \Omega$ with the beam pipe wall that bulges out. The longitudinal and transverse coupling impedances have been calculated to be $[3]$

$$
\begin{gathered}
Z_{\|}=2 M Z_{c}\left(\frac{\phi_{0}}{2 \pi}\right)^{2}\left(\sin ^{2} \frac{\omega \ell}{c}+j \sin \frac{\omega \ell}{c} \cos \frac{\omega \ell}{c}\right), \\
Z_{\perp}=\frac{c}{2 b^{2}}\left(\frac{4}{\phi_{0}}\right)^{2} \sin ^{2} \frac{\phi_{0}}{2} \frac{Z_{\|}}{\omega}
\end{gathered}
$$

where the factor $\frac{1}{2}$ is inserted in the expression for $Z_{\perp}$ because one half of the BPM sets work for the horizontal and one half for the vertical. At low frequencies, the impedances are inductive,

$$
\begin{gathered}
\frac{Z_{\|}}{n}=j 2 M Z_{c}\left(\frac{\phi_{0}}{\pi}\right)^{2} \frac{\ell}{R}=j 0.363 \Omega, \\
Z_{\perp}=j 0.431 \mathrm{M} \Omega / \mathrm{m} .
\end{gathered}
$$


At high frequencies, the reactive parts of the impedances oscillate between inductive and capacitive; for example, the first zero occurs when $f=c /(2 \ell)=0.833 \mathrm{GHz}$. The real parts rise from zero quadratically with frequency and $\mathcal{R} e Z_{\|}$has a peak value of $2.02 \mathrm{k} \Omega$ at $0.833 \mathrm{GHz}$, or $\mathcal{R} e Z_{\|} / n=0.116 \Omega$.

\section{II.4 BELLOWS}

There are about 1000 bellows in the Tevatron, each of which consists of 24 convolutions of width $1.04 \mathrm{~mm}$ between inner and outer radii of 3.94 and $4.58 \mathrm{~cm}$ as shown in Fig. 2. We run ABCI [4] to obtain the wakes of azimuthal modes $m=0$ and $m=1$, from which the longitudinal and transverse impedances are computed and plotted in Figs. 3 and 4. We see that there is a broad-band peaks centered around $7.0 \mathrm{GHz}$ with $Q \approx 2$ and shunt impedance $R_{s} \approx 100 \Omega$ (per bellows). This gives for 1000 bellows a broad-band which peaks at $\operatorname{Re} Z_{\|} / n \approx 0.68 \Omega$ and an inductive part $\operatorname{Im} Z_{\|} / n \approx R_{s} /\left(Q n_{r}\right) \approx 0.34 \Omega$.

For the transverse impedance in Fig. 4, there is also a broad-band peak around $7.0 \mathrm{GHz}$ with $Q \approx 0.73$ and shunt impedance $R_{s} \approx 1.5 \mathrm{k} \Omega / \mathrm{m}$ (per bellows), or $\operatorname{Re} Z_{\perp} \approx 1.1 \mathrm{M} \Omega / \mathrm{m}$ for the whole ring. Below $\sim 2 \mathrm{GHz}$, the reactive part of the impedance is $\operatorname{Im} Z_{\perp} \approx 0.40 \mathrm{M} \Omega / \mathrm{m}$.

There are also sharp resonances. We believe, however, that they will be present at slightly different frequencies for different bellows. Therefore, it is reasonable to expect them to add up to broader resonances instead, but with much smaller areas under the impedance curves than the broad-bands at $7.0 \mathrm{GHz}$ for both the longitudinal and transverse impedances.

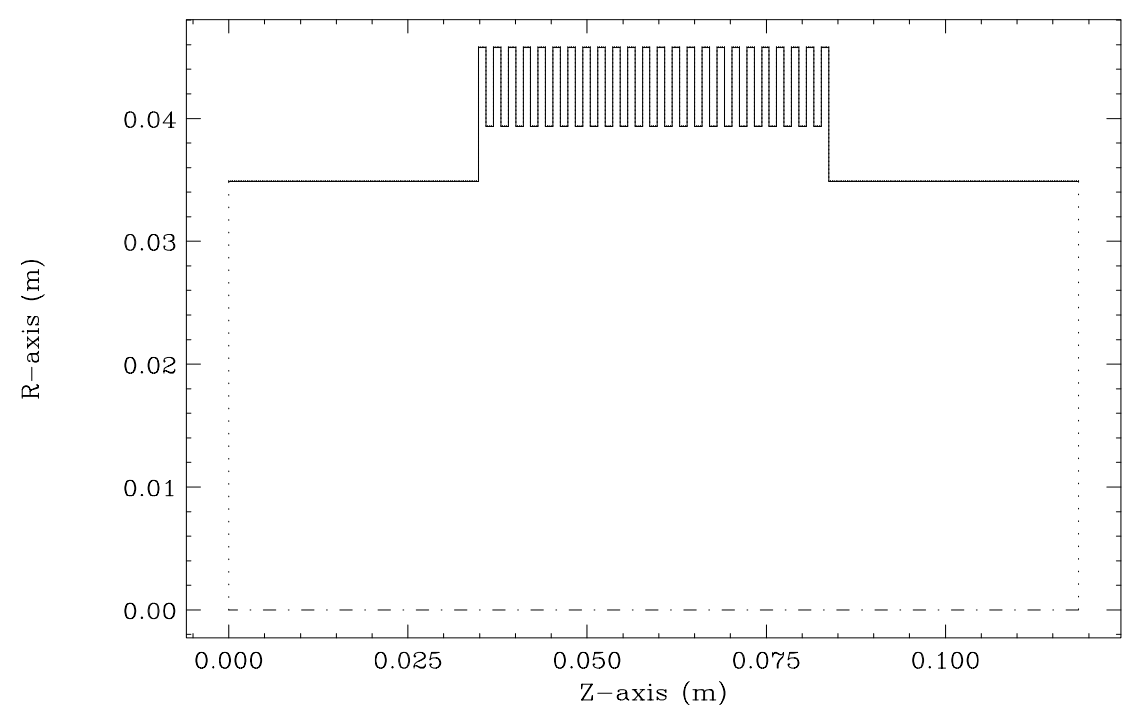

Figure 2: A model of one Tevatron bellows used in ABCI. 


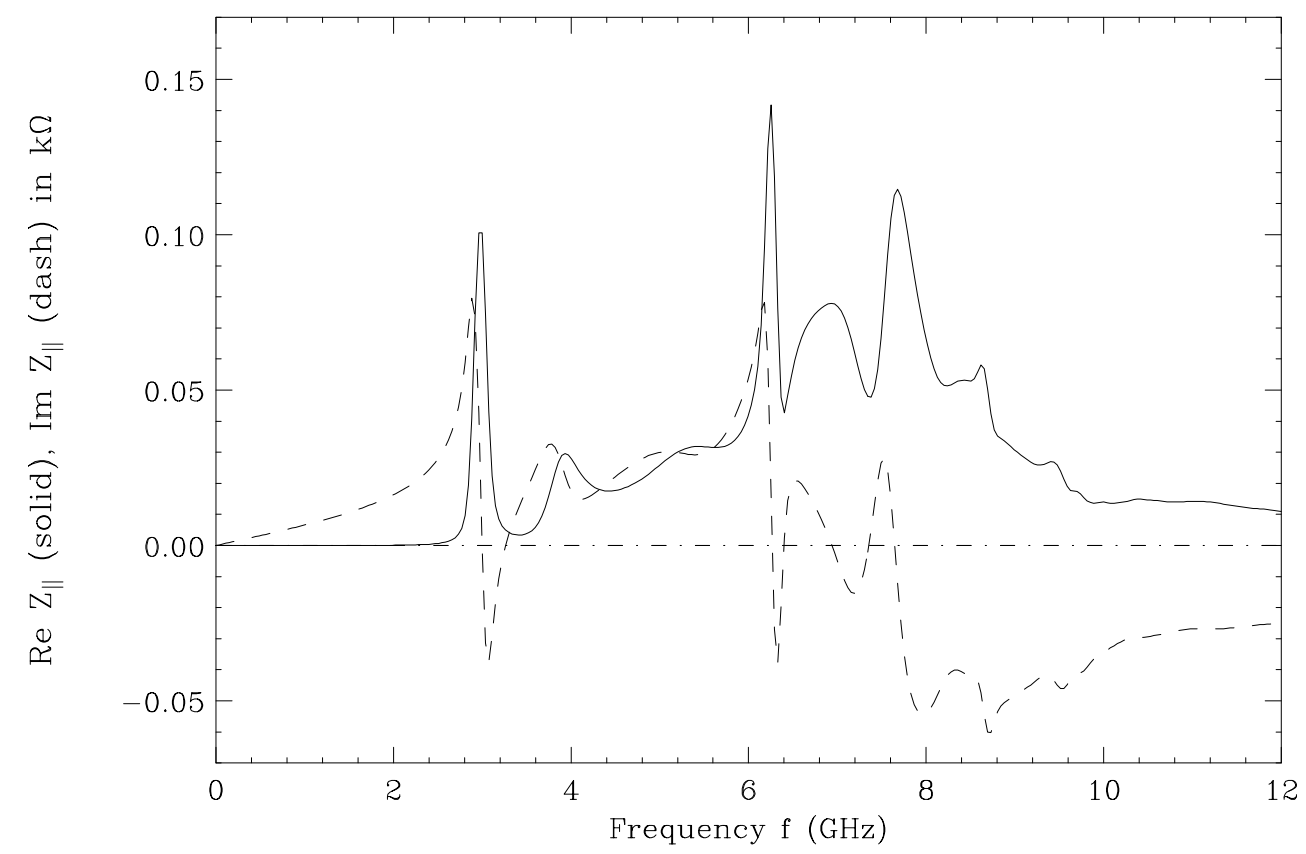

Figure 3: The real and imaginary parts of $Z_{\|}$in a Tevatron bellows as computed by ABCI.

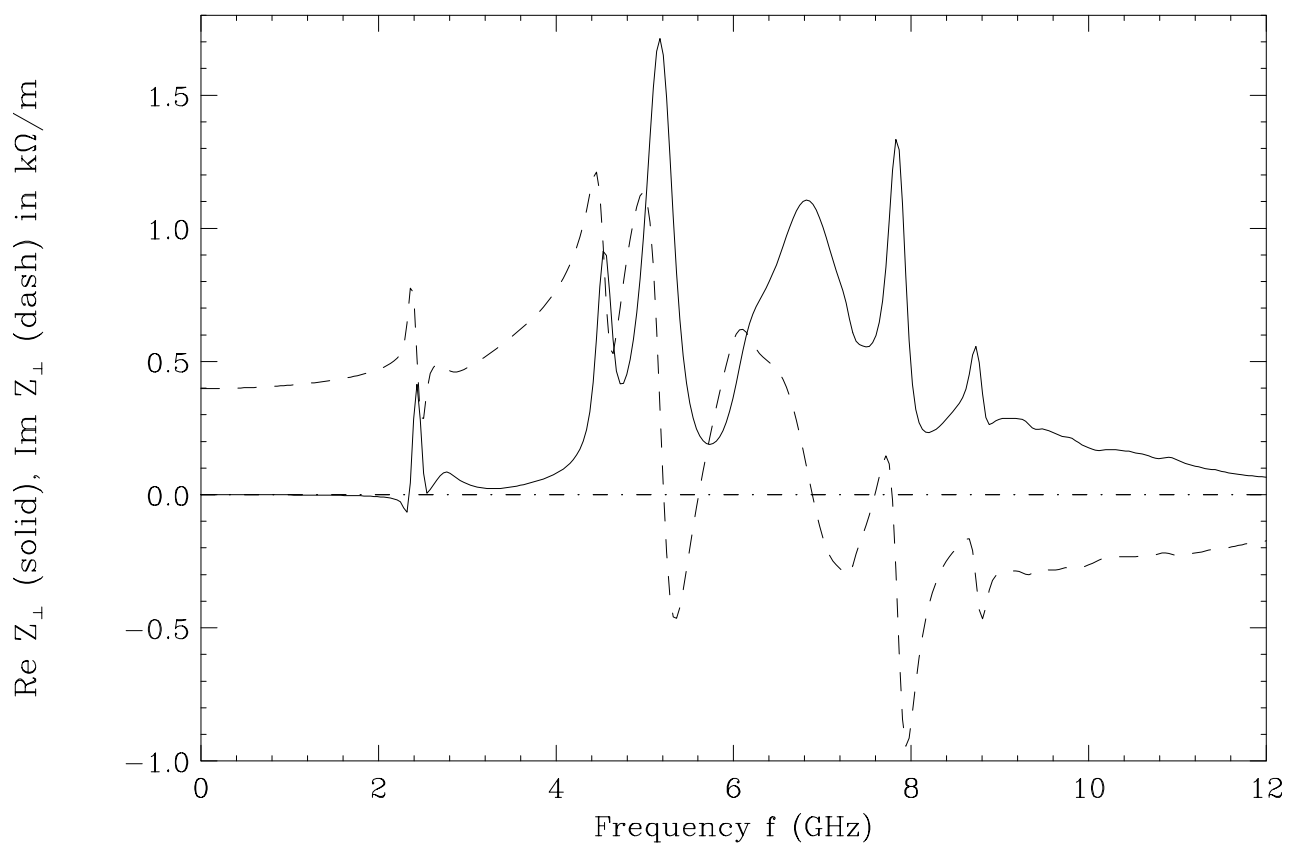

Figure 4: The real and imaginary parts of $Z_{\perp}$ in a Tevatron bellows as computed by ABCI. 


\section{II.5 SEPARATORS}

There are 11 electrostatic separators in the Tevatron vacuum chamber. Their function is to separate the proton and antiproton bunches so that they will not collide with each other except at designated interaction points. We use the MAFIA code [5] to compute the wake potentials left by a short bunch for both the monopole and dipole modes. Because of the limitation on number of grid points of the code, it is impossible to input the exact details of the separators. Instead, we model a separator system as two plates $20 \mathrm{~cm}$ wide and $2.57 \mathrm{~m}$ long inside a circular cavity chamber of length $2.75 \mathrm{~m}$ and radius $18 \mathrm{~cm}$ as illustrated in Fig. 5 . The beam pipe is circular in cross section with radius $4 \mathrm{~cm}$. The grid size is $1 \mathrm{~cm}$ in the longitudinal and horizontal directions, but $1.125 \mathrm{~cm}$ in the vertical direction. Next the Fourier transforms are computed to arrive at the longitudinal monopole and impedance and transverse dipole impedance, which are plotted in Fig. 6 and 7 up to $3 \mathrm{GHz}$ [6]. We believe that this simplified model will retain all the essential features of the impedances.
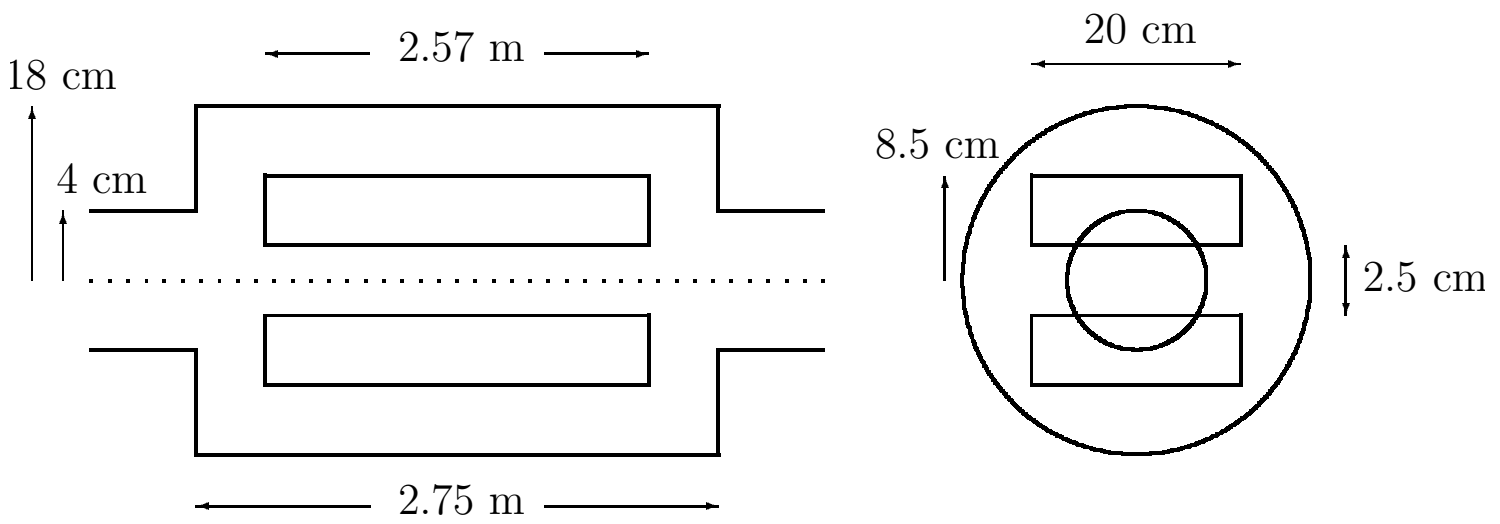

Figure 5: The simplified separator system used in MAFIA computation of monopole and dipole wake potentials.

The separator system can be viewed as two pill-box cavities joined by a transmission line. For a closed-end pill-box cavity of radius $18 \mathrm{~cm}$ the first few monopoles resonances are at $f_{010}=0.637 \mathrm{GHz}$, $f_{020}=1.46 \mathrm{GHz}, f_{030}=2.29 \mathrm{GHz}, \cdots$, and the first few dipoles resonances are at $f_{110}=1.02 \mathrm{GHz}$, $f_{120}=1.86 \mathrm{GHz}, f_{130}=2.70 \mathrm{GHz}, \cdots$. Actually these resonances are seen at $0.75,1.51$, and $2.24 \mathrm{GHz}$ in Fig. 6 and 1.23, 1.80, and $2.74 \mathrm{GHz}$ in Fig. 7. The shifts are probably due to the fact the the cavities are not closed. These modes are below the cutoff frequency of $2.87 \mathrm{GHz}$ for the $4 \mathrm{~cm}$-radius beam pipe. However, some resonances are very much broadened. We believe that this is a result of the transmission effect between the two cavities. We see from Figs. 6 and 7 that the 11 separators will give below $\sim 0.6 \mathrm{GHz}$ the contributions $\operatorname{Im} Z_{\|} / n=0.21 \Omega$ and $\operatorname{Im} Z_{\perp}=0.82 \mathrm{M} \Omega / \mathrm{m}$, which are not too small. 


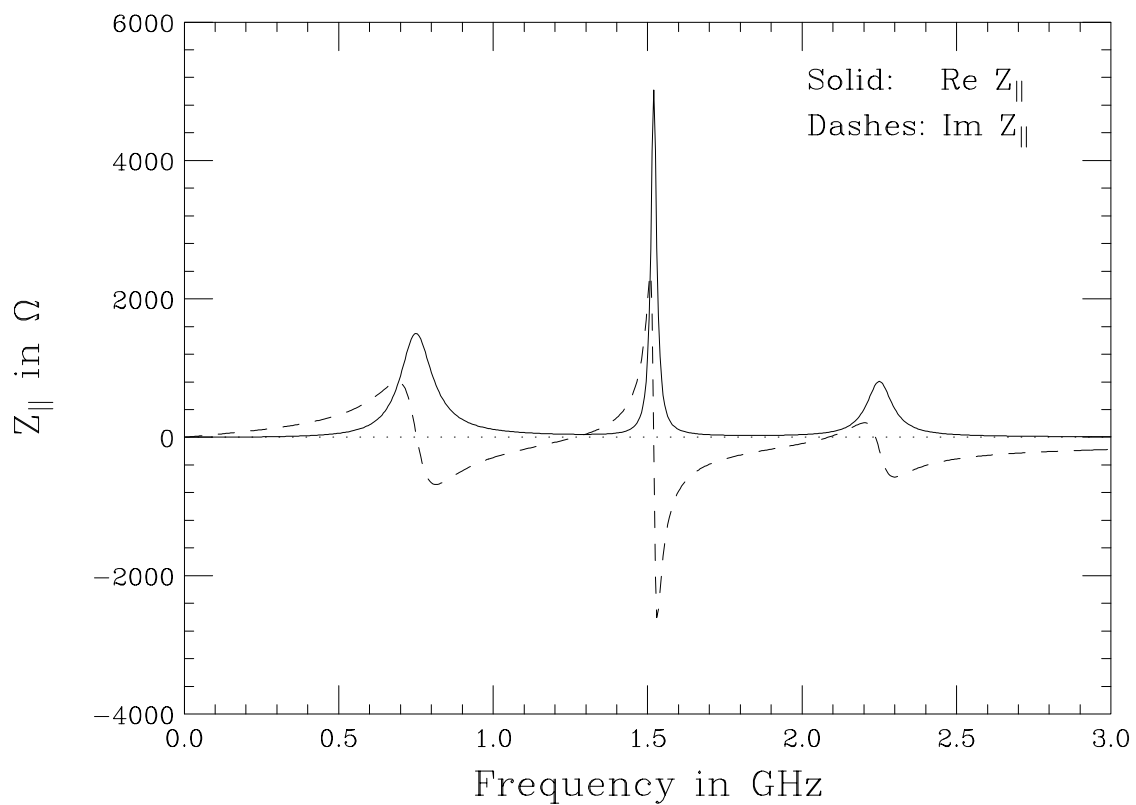

Figure 6: The real and imaginary parts of the longitudinal impedance $Z_{\|}$of one separator system.

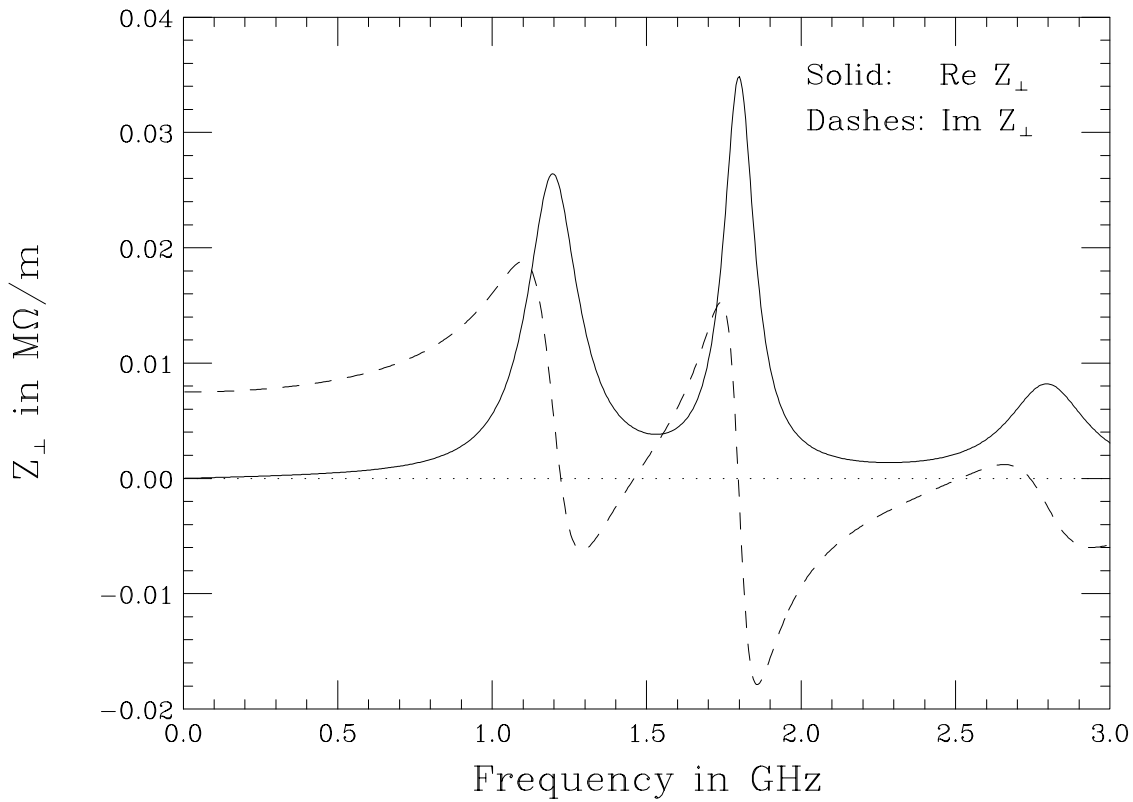

Figure 7: The real and imaginary parts of the vertical impedance $Z_{\perp}$ of one separator system. 


\section{II.6 RF CAVITIES}

Some higher-order monopole modes of a Tevatron rf cavity have been measured by Sun and Colestock [7] in 1995 using both the method of dielectric bead-pull and wire measurement. The resonances quoted in Table II are based on bead measurements only, as the modes with wire present were shifted in frequency so much to permit positive identification of the modes. A combination of dielectric beads, metallic beads and needles was used to perturb the cavity. The ultimate accuracy was determined most likely by temperature drifts in either the cavity or the network analyzer to about 0.5 degrees, corresponding to impedances (depending on their $Q$ values) to a few $\mathrm{k} \Omega$. We also use the URMEL code [8] to compute some lower modes and the results are listed also in Table II for comparison. We find that the URMEL resonant frequencies and $R / Q$ for these modes agree rather well with Sun's measurement. On the other hand, the quality factors $Q$ do not agree so well. This may be because URMEL computes the modes of the bare cavity, while some of these modes have actually been de-Qued passively. Also there are a lot of structures inside the cavity and these structures have not been included in the simplified model of the cavity used in URMEL computation.

Table II: Longitudinal modes for one whole cavity.

\begin{tabular}{|c|c|c|c|c|c|c|}
\hline \multirow[b]{2}{*}{ Mode Type } & \multicolumn{3}{|c|}{ URMEL Results } & \multicolumn{3}{|c|}{ Sun's Measurements } \\
\hline & $\begin{array}{r}\text { Frequency } \\
(\mathrm{MHz})\end{array}$ & $\begin{array}{r}R / Q \\
(\Omega)\end{array}$ & $Q$ & $\begin{array}{r}\text { Frequency } \\
(\mathrm{MHz})\end{array}$ & $\begin{array}{r}R / Q \\
(\Omega)\end{array}$ & $Q$ \\
\hline TM0-EE-1 & 53.49 & 87.65 & 9537 & 53.11 & 109.60 & 6523 \\
\hline TM0-ME-1 & 84.10 & 22.61 & 12819 & 56.51 & 18.81 & 3620 \\
\hline TM0-EE-2 & 166.56 & 18.47 & 16250 & 158.23 & 11.68 & 6060 \\
\hline TM0-ME-2 & 188.94 & 10.83 & 18235 & & & \\
\hline TM0-EE-3 & 285.94 & 7.53 & 20524 & 310.68 & 7.97 & 15923 \\
\hline TM0-ME-3 & 308.46 & 4.07 & 22660 & & & \\
\hline TM0-EE-4 & 402.69 & 4.93 & 25486 & 439.77 & 5.23 & 13728 \\
\hline TM0-ME-4 & 431.34 & 1.72 & 26407 & 424.25 & 1.28 & 6394 \\
\hline TM0-EE-5 & 511.69 & 5.57 & 25486 & 559.48 & 6.73 & 13928 \\
\hline \multirow[t]{3}{*}{ TM0-ME-5 } & 549.57 & 1.36 & 29453 & & & \\
\hline & & & & 748.18 & 10.90 & 13356 \\
\hline & & & & 768.03 & 2.47 & 16191 \\
\hline
\end{tabular}

There have not been any measurement for the dipole modes. Therefore, we need to rely on the URMEL results, which are listed in Table III. Except for the fundamental, we believe that all these higher-order modes will have frequencies varied slightly from cavity to cavity. Therefore, we expect them to be broadened or the quality factors lowered when all the rf cavities of the Tevatron are considered. 
Table III: Transverse modes for one whole cavity.

\begin{tabular}{lcrc}
\hline Mode Type & $\begin{array}{c}\text { Frequency } \\
(\mathrm{MHz})\end{array}$ & $\begin{array}{c}R / Q \\
(\Omega / \mathrm{m})\end{array}$ & $Q$ \\
\hline 1-EE-1 & 486.488 & 229.80 & 31605 \\
1-ME-2 & 486.864 & 148.95 & 31487 \\
1-EE-2 & 513.370 & 117.38 & 33262 \\
1-ME-3 & 518.317 & 117.93 & 34008 \\
1-EE-3 & 561.727 & 81.62 & 33029 \\
1-ME-4 & 575.298 & 3.84 & 35810 \\
1-EE-4 & 625.123 & 61.00 & 32598 \\
1-ME-5 & 650.853 & 35.21 & 37592 \\
1-EE-5 & 699.723 & 54.76 & 33407 \\
\hline
\end{tabular}

\section{II.7 SUMMARY}

We try to add up the individual impedances studied in the previous sections and arrive at the total in Figs. 8 and 9. The impedances are plotted as functions of revolution harmonics and also frequencies. For the contributions of the resistive wall and Lambertson magnets to the transverse impedance, the residual betatron tune in Eqs. (2.5), (2.12), and (2.14) has been set to zero. Since logarithmic scales have been used, only the positive-frequency parts of the impedances are plotted and the capacitive parts of the impedances are not shown. The higher-order modes of the cavities have not been included, because they are too narrow to be visible in log-log plots. The impedances of the 11 separators are included, although they have not been plotted separately in order not to make the figures too crowded.

We see that the resistive wall and the Lambertsons dominate mostly below $\sim 10 \mathrm{MHz}$. Then the contributions of the bellows and BPM's are clearly seen in the region of $10 \mathrm{MHz}$ to $\sim 1 \mathrm{GHz}$. The peaks near $1 \mathrm{GHz}$ are the resonances of the separators. Finally, there are the broad resonances of the bellows at $\sim 7 \mathrm{GHz}$. Notice that the sharper resonances of the bellows around 2 to $3 \mathrm{GHz}$ in Figs. 3 and 4 do not show up in these plots. This is because the increment in frequency in the logarithmic scale has not been fine enough. There are other contributions to the inductive impedances such as steps in the vacuum chamber, kickers, etc. Therefore, it will be reasonable if we add $\sim 1$ to $2 \Omega$ and $\sim 1$ to $2 \mathrm{M} \Omega / \mathrm{m}$, respectively, to the longitudinal impedance per harmonic and transverse impedance around beam-pipe cutoff, which, for a square beam pipe of side $h=6 \mathrm{~cm}$, is roughly $f_{\text {cutoff }} \approx c /(2 h)=2.5 \mathrm{GHz}$. Thus, around $f_{\text {cutoff }}$, the longitudinal impedance per harmonic and transverse impedance are roughly $1.8 \Omega$ and $2.0 \mathrm{M} \Omega / \mathrm{m}$, respectively. The proton bunch has a rms bunch length of $\sigma_{\ell}=37 \mathrm{~cm}$ which is very much larger than the radius of the beam pipe. The longest wavelength $\lambda$ that can perturb the bunch is roughly two times the total bunch length, or $\lambda \approx 4 \sqrt{6} \sigma_{\ell}=3.63 \mathrm{~m}$. Thus we can define a bunch cutoff frequency as $f_{c} \approx c / \lambda=82.8 \mathrm{MHz}$. At this frequency, $\operatorname{Re} Z_{\|} / n \approx \operatorname{Im} Z_{\|} / n \approx 3.0 \Omega$ and $\operatorname{Re} Z_{\perp} \approx \operatorname{Im} Z_{\perp} \approx 3.0 \mathrm{M} \Omega / \mathrm{m}$. 


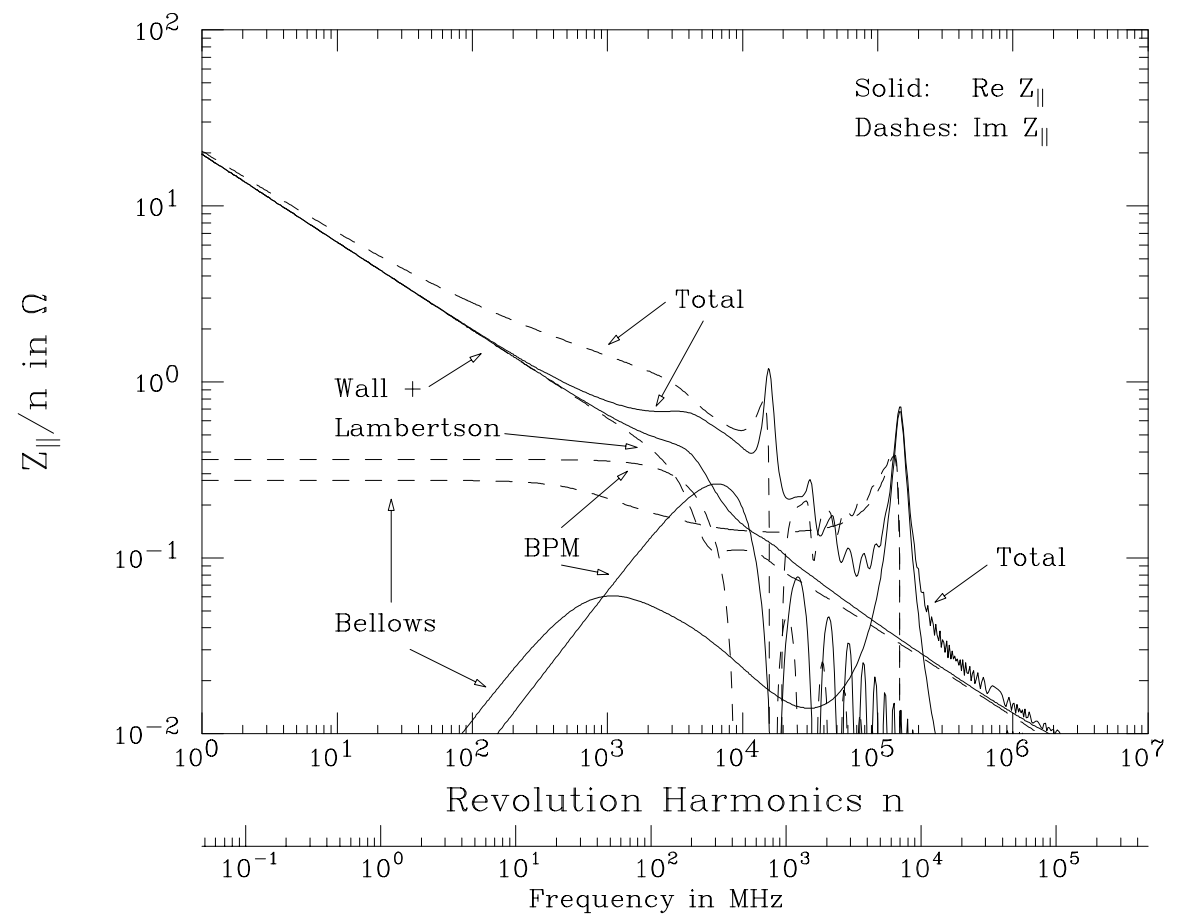

Figure 8: The real and imaginary parts of $Z_{\|} / n$ contributions to the Tevatron vacuum chamber. The capacitive parts are not shown.

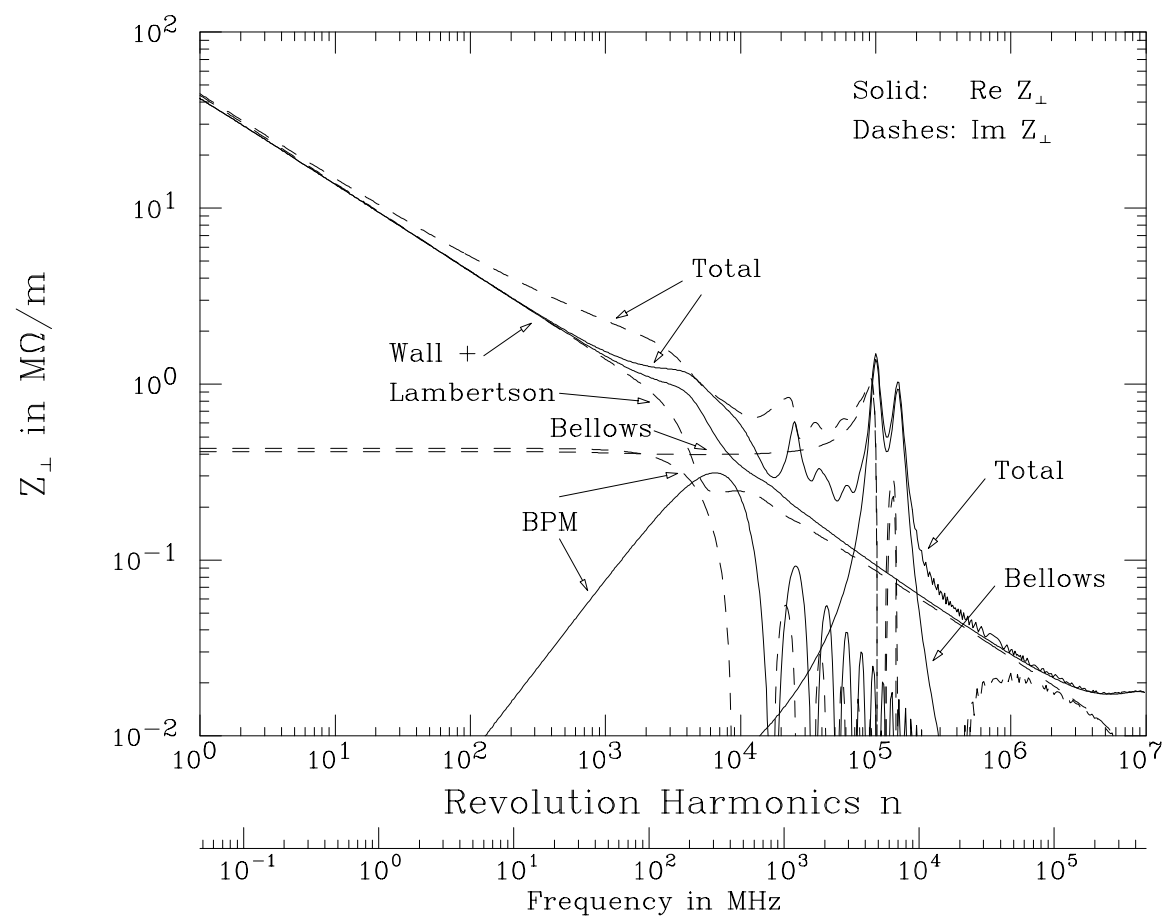

Figure 9: The real and imaginary parts of $Z_{\perp}$ contributions to the Tevatron vacuum chamber. The capacitive parts are not shown. 


\section{POTENTIAL-WELL DISTORTION}

The proton or antiproton bunches will see an rf voltage of $V_{\mathrm{rf}}=1 \mathrm{MV}$ per turn, implying a coherent synchrotron tune of $\nu_{s 0}=7.077 \times 10^{-4}$ at $1 \mathrm{TeV}$. For a bunch of rms length $\sigma_{\tau_{0}}=1.234 \mathrm{~ns}$, the rms momentum spread is therefore

$$
\sigma_{\delta_{0}}=\frac{\omega_{0} \sigma_{\tau_{0}} \nu_{s 0}}{\eta}=9.262 \times 10^{-5}
$$

where $\omega_{0}=2 \pi f_{0}$ is the angular revolution frequency. Assuming a parabolic bunch distribution, the half bunch length is $\hat{\tau}_{0}=\sqrt{5} \sigma_{\tau_{0}}=2.760 \mathrm{~ns}$ and the half momentum spread is $\hat{\delta}_{0}=\sqrt{5} \sigma_{\delta_{0}}=2.071 \times 10^{-4}$. Therefore the bunch area is $S=5 \pi \sigma_{\tau_{0}} \sigma_{\delta_{0}} E=1.796 \mathrm{eV}$-s. It is worthwhile to point out that the bunch area appears to be smaller than the actual Tevatron bunch area measured at injection. This is because once the rms is given, the bunch area depends very much on the bunch distribution one prefers. There are no tails in the parabolic distribution, the bunch area is therefore smaller. This can be thought of the area of the core part of an actual bunch. For the cosine-square distribution $\rho(\tau)=\cos ^{2} \pi \tau /(2 \hat{\tau}) / \hat{\tau}$, we have $\left(\hat{\tau} / \sigma_{\tau}\right)^{2}=3 \pi^{2} /\left(3 \pi^{2}-6\right)=7.65$ and the total bunch area will be much larger. On the other hand, the bunch area of a bi-Gaussian distribution encircling $95 \%$ of the bunch particles is $S_{95 \%}=6 \pi \sigma_{\tau_{0}} \sigma_{\delta_{0}} E=1.796 \mathrm{eV}-\mathrm{s}$ In this section, we prefer the parabolic distribution because it makes the analysis much simpler.

In the presence of an inductive part of the longitudinal impedance, the bunch will be lengthened to $\hat{\tau}=k \hat{\tau}_{0}$ above transition, and the momentum spread diminished to $\hat{\delta}=\hat{\delta}_{0} / k$ so that the bunch area remains constant. The lengthening ratio $k$ satisfies the quartic equation [9]

$$
1=k^{4}-k D,
$$

where

$$
D=\left.\frac{3 e N_{p}}{2 \omega_{0}^{2} h V_{\mathrm{rf}} \cos \phi_{s} \hat{\tau}_{0}^{3}} \frac{Z_{\|}}{n}\right|_{\text {ind }},
$$

and $\phi_{s}$ is the synchronous angle which we take as zero here. We find that the lengthening ratios are $k=1.015$, $1.023,1.030$, and 1.038 , respectively, when the inductive part of the impedance per harmonic $Z_{\|} /\left.n\right|_{\text {ind }}=2$, 3,4 , and $5 \Omega$. The Tevatron bunch spectrum has a rms frequency of $1 /\left(2 \pi \sigma_{\tau}\right) \approx 130 \mathrm{MHz}$. From Fig. 8 , it is reasonable to assume $Z_{\|} /\left.n\right|_{\text {ind }} \approx 2$ to $3 \Omega$. Thus the amount of bunch lengthening will not be appreciable. The longitudinal impedance does have a real part which is of the same order of magnitude as the reactive part. The real part will lead to a left-right asymmetric distortion, which we think would be small also.

The potential-well distortion can have other consequences. Usually we measure the total bunch length $2 \hat{\tau}$ and infer the half momentum spread $\hat{\delta}$ and bunch area $S$ according to

$$
\hat{\delta}=\frac{\omega_{0} \nu_{s} \hat{\tau}}{\eta} \quad \text { and } \quad S=\frac{\pi E \omega_{0} \nu_{s} \hat{\tau}^{2}}{\eta} .
$$

Because of the defocusing effect of the inductive impedance above transition, the incoherent synchrotron tune $\nu_{s}$ will be less than the coherent synchrotron tune $\nu_{s 0}$. Comparing with Eq. (3.1), they are related by

$$
\nu_{s}=\frac{\nu_{s 0}}{k^{2}} .
$$

Thus the effective rf voltage becomes

$$
V_{\mathrm{rf}_{\mathrm{eff}}}=\frac{V_{\mathrm{rf}}}{k^{4}} .
$$


Usually the incoherent synchrotron tune is difficult to measure. If one substitutes the coherent synchrotron tune into Eq. (3.4), one would have estimated the momentum spread and bunch area too big by the factor $k^{2}$. This will give a wrong idea about the amount of Landau damping.

\section{LONGITUDINAL MICROWAVE INSTABILITY}

The beam current at a revolution harmonic $n$ interacts with the longitudinal coupling impedance of the vacuum chamber at the same harmonic to create a bucket at that harmonic and the beam particles are bunched. This phenomenon of self-bunching is called longitudinal microwave instability. This bunching or growth will not take place if the spread in revolution frequency among the beam particles is large enough. Applying to a bunch, we have the Boussard-modified Keil-Schnell stability criterion on the coupling impedance $[10,11]$ :

$$
\left|\frac{Z_{\|}}{n}\right|<F \frac{\eta E}{e I_{\text {peak }}} \delta_{\text {FWHM }}^{2}
$$

For a parabolic bunch, the form factor $F \approx 1, \delta_{\mathrm{FWHM}}=\sqrt{2} \hat{\delta}$, and the peak current $I_{\text {peak }}=3 I_{b} /\left(\left(4 \hat{\tau} f_{0}\right)\right.$ with $I_{b}$ being the average bunch current. The above can also be written as

$$
\left|\frac{Z_{\|}}{n}\right|<\frac{16 \pi}{3 I_{b}}\left(\omega_{0} \hat{\tau}\right)^{3} h V_{\mathrm{rf}_{\mathrm{eff}}},
$$

or

$$
\left|\frac{Z_{\|}}{n}\right|<\frac{8.2^{1 / 4}}{3 \pi^{5 / 4} I_{b}}\left(f_{0} S / e\right)^{3 / 2}\left(\frac{\eta}{E / e}\right)^{3 / 4}\left(h V_{\mathrm{rf}_{\mathrm{eff}}}\right)^{1 / 4}
$$

Therefore if the bunch area $S$ and momentum spread $\hat{\delta}$ are inferred from Eq. (3.4) using the coherent synchrotron tune, and the effective rf voltage $V_{\mathrm{rf}_{\mathrm{eff}}}$ is replaced by the unperturbed $V_{\mathrm{rf}}$ displayed in the oscilloscope, one needs to divide the right sides of Eqs. (4.1) to (4.3) by the 4th power of the potential-well bunch lengthening factor $k$ defined in Sec. III. For a fixed unperturbed $V_{\mathrm{rf}}=1 \mathrm{MV}$, and half bunch length $37 \mathrm{~cm}$, the stability limit is most stringent at the storage energy of $E=1 \mathrm{TeV}$ and is given in Table IV for various assumption of the inductive part of the impedance per harmonic $Z_{\|} /\left.n\right|_{\text {ind }}$.

Table IV: Bunch lengthening ratio $k$ and longitudinal microwave stability limits at $E=1 \mathrm{TeV}$ versus the inductive part $Z_{\|} /\left.n\right|_{\text {ind }}$.

\begin{tabular}{ccc}
\hline$\left.\mathcal{I} m \frac{Z_{\|}}{n}\right|_{\text {ind }}$ & $k$ & $\left|\frac{Z_{\|}}{n}\right|$ limit \\
\hline $0 \Omega$ & 1.000 & $20.63 \Omega$ \\
$1 \Omega$ & 1.008 & $20.01 \Omega$ \\
$2 \Omega$ & 1.015 & $19.41 \Omega$ \\
$3 \Omega$ & 1.023 & $18.84 \Omega$ \\
$4 \Omega$ & 1.030 & $18.30 \Omega$ \\
$5 \Omega$ & 1.039 & $17.78 \Omega$ \\
\hline
\end{tabular}


Microwave instability is essentially a coasting beam effect and self-bunching must occur much faster than a synchrotron oscillation, otherwise the growth will decohere. Therefore the perturbation should have a half-wavelength less than the length of the bunch, or a frequency $f>1 /(4 \hat{\tau})=90.6 \mathrm{MHz}$. From Fig. 8, together with a generous allowance for other contributions not included, $\left|Z_{\|} / n\right|$ of the Tevatron vacuum chamber will be at most a few ohms, which is very much below the the Keil-Schnell limit listed in Table IV. Thus the longitudinal microwave instability should not pose any problem in Run II.

\section{LONGITUDINAL COUPLED BUNCH INSTABILITIES}

The long-range wake left by the higher-order resonant modes of the rf cavities may couple the longitudinal motions of the bunches in the Tevatron. Assuming $M$ bunches of equal intensity equally spaced in the ring, there are $\mu=0,1, \cdots, M-1$ modes of oscillations in which the center-of-mass of a bunch lags behind its predecessor by the phase $2 \pi \mu / M$. In addition, an individual bunch in the $\mu$-th coupled-bunch mode can oscillate in the synchrotron phase space about its center-of-mass in such a away that there are $m=1,2, \cdots$ nodes along the bunch longitudinally (not including the ends). For example, $m=1$ is the rigid dipole mode, where the bunches move rigidly as they execute synchrotron oscillations, $m=2$ is the quadrupole mode where the bunch head and tail oscillate longitudinally $180^{\circ}$ out of phase. Actually, this has been a simplified description of the modes of perturbation inside a bunch. The full description involves two eigen-numbers, for example, the the azimuthal and the radial.

If the driving narrow resonance falls on a $\mu$-th coupled bunch line, Sacherer's growth rate for the $m$ th mode is [12]

$$
\frac{1}{\tau_{m \mu}}=\frac{e \eta M I_{b} R_{s} f_{0}}{2 \pi E \nu_{s} B_{0}} D F_{m}(\Delta \phi),
$$

where $B_{0}=\tau_{L} f_{0}$ is the single-bunch bunching factor with $\tau_{L}=2 \hat{\tau}$ being the total bunch length, $\nu_{s}$ is the perturbed synchrotron tune, $R_{s}$ is the shunt impedance of the sharp driving resonance at frequency $f_{r}=\omega_{r} /(2 \pi)$. The factor $D$ is a function of the decay decrement $\alpha \tau_{\text {sep }}$ between successive bunches, where $\alpha=\omega_{r} /(2 Q)$ is the HWHM of the resonance of quality factor $Q$ and $\tau_{\text {sep }}$ is the bunch separation. It is defined as

$$
D\left(\alpha \tau_{\text {sep }}\right)=-i 2 \alpha \tau_{\text {sep }} \sum_{k=0}^{\infty} e^{-2 \pi i k \mu / M-k(\alpha-i \Omega) \tau_{\text {sep }}} \sin k \omega_{r} \tau_{\text {sep }},
$$

The maximum magnitude of $D$ is shown in Fig. 10. The form factor for parabolic bunches is given by

$$
F_{m}(\Delta \phi)=\frac{16 m}{\Delta \phi}\left[J_{m}^{2}\left(\frac{1}{2} \Delta \phi\right)-J_{m+1}\left(\frac{1}{2} \Delta \phi\right) J_{m-1}\left(\frac{1}{2} \Delta \phi\right)\right],
$$

where $\Delta \phi=2 \pi f_{r} \tau_{L}$ is the phase change of the resonator during the bunch passage from head to tail, and is plotted in Fig. 11. Note that mode $m$ peaks roughly at $\Delta \phi=m \pi$. This is reasonable because, as was mentioned above, mode $m$ represents a longitudinal variation along the bunch with $m$ nodes (not including the ends) and it will be most easily excited when the bunch sees a phase variation of $m \pi$ of the driving resonance as it passes through the cavity gap from head to tail. Note that $F_{m}$ decreases as $m$ increases, implying that the higher $m$ modes will not be excited so easily.

The rf voltage during the whole ramp is about $1 \mathrm{MV}$. Therefore the growth will be most severe at the 


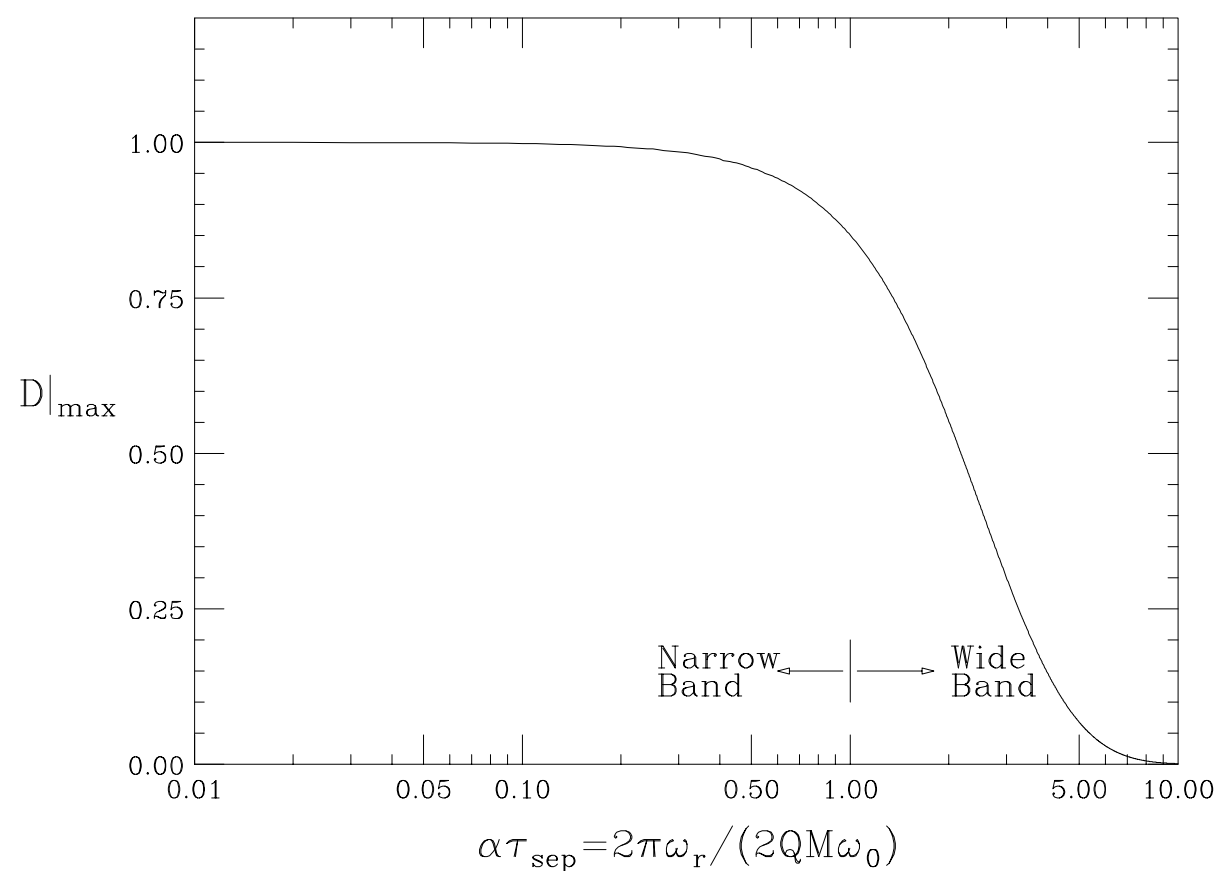

Figure 10: $|D|_{\max }$ as a function of bunch-to-bunch decay decrement $\alpha \tau_{\text {sep. }}$. Note that $|D|_{\max } \approx 1$ for narrow resonances but drops very rapidly as the resonance becomes broader.

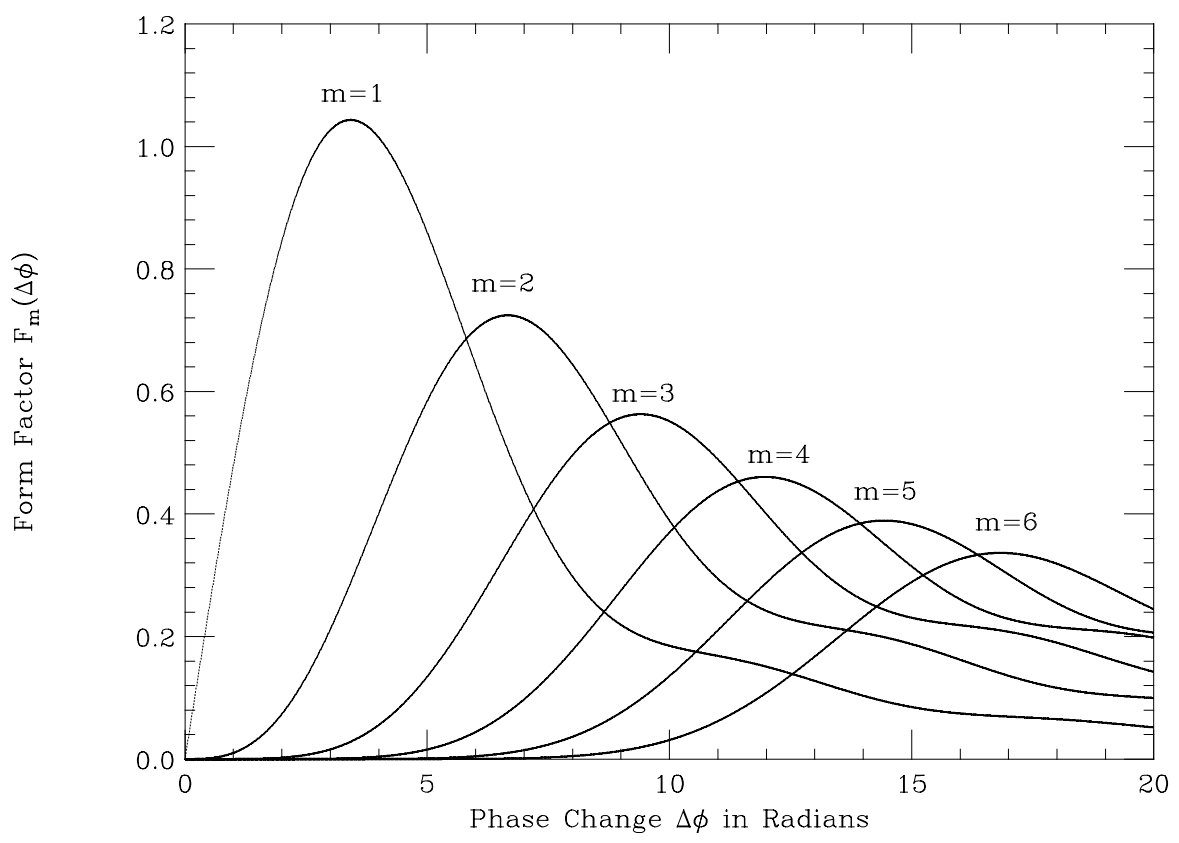

Figure 11: Form factor for longitudinal oscillation inside a bunch with $m=$ $1,2,3,4,5$ and 6 nodes. 
injection energy of $E=150 \mathrm{GeV}$. The growth rates for the first few modes are listed in Table $\mathrm{V}$ for the $36 \times 36$ scenario.

Table V: Longitudinal coupled-bunch growth rates driven by the higher-order modes of the rf cavities at injection for the $36 \times 36$ scenario in Run II.

\begin{tabular}{crrrrrrrrr}
\hline$f_{r}$ & $R_{s}$ & $Q$ & \multicolumn{1}{c}{$\Delta \phi$} & \multicolumn{6}{c}{ Growth Rate in sec $\mathrm{sen}^{-1}$} \\
$\mathrm{MHz}$ & $\mathrm{k} \Omega$ & & $\mathrm{rad}$ & $m=1$ & $m=2$ & $m=3$ & $m=4$ & $m=5$ & $m=6$ \\
\hline 56.5 & 68 & 3620 & 0.88 & $\underline{0.606}$ & 0.010 & 0.000 & 0.000 & 0.000 & 0.000 \\
158.2 & 70 & 6060 & 2.45 & $\underline{1.415}$ & 0.189 & 0.009 & 0.000 & 0.000 & 0.000 \\
310.7 & 124 & 15923 & 4.82 & $\underline{\underline{2.329}}$ & $\underline{1.443}$ & 0.305 & 0.033 & 0.002 & 0.000 \\
424.2 & 8 & 6394 & 6.58 & $\underline{\underline{0.089}}$ & 0.124 & 0.056 & 0.012 & 0.001 & 0.000 \\
439.8 & 71 & 13728 & 6.82 & $\underline{0.714}$ & $\underline{1.089}$ & 0.542 & 0.129 & 0.018 & 0.002 \\
559.5 & 93 & 13928 & 8.68 & $\underline{0.469}$ & $\underline{1.103}$ & $\underline{1.071}$ & 0.478 & 0.120 & 0.019 \\
748.2 & 145 & 13356 & 11.60 & $\underline{0.484}$ & $\underline{0.789}$ & $\underline{1.333}$ & $\underline{1.397}$ & 0.787 & 0.269 \\
768.0 & 39 & 16191 & 11.91 & $\underline{0.128}$ & 0.206 & 0.342 & 0.386 & 0.236 & 0.087 \\
\hline \multicolumn{4}{l}{ Laudau Damping rate $\left(\mathrm{s}^{-1}\right)$} & 0.000 & 0.555 & 0.679 & 0.784 & 0.877 & 0.961 \\
\hline
\end{tabular}

These higher-order modes were measured by Sun [7] in 1995 using the method of dielectric bead pull. Here, we assume that the peak of each resonance is at exactly a synchrotron line on the left side of the revolution harmonic. Also, the higher-order resonances of each cavity will not be at exactly the same frequency. In other words, for all the 8 cavities, we assume the resonances will be de-Qued 8 times. Therefore, for each mode, the shunt impedance of one cavity has been used in Eq. (5.1) when the computation is performed.

The spread of the synchrotron frequency due to the nonlinear sinusoidal rf wave form can be written as

$$
\frac{\Delta \omega_{s}}{\omega_{s}}=\frac{2}{3}\left(\frac{1+\Gamma^{2}}{1-\Gamma^{2}}\right)\left(\frac{h \tau_{L} f_{0}}{2}\right)^{2}=0.0143 \text { or } \Delta f_{s}=1.25 \mathrm{~Hz}
$$

when the nominal synchrotron tune $\nu_{s}=1.83 \times 10^{-3}$ is assumed at the injection energy of $150 \mathrm{GeV}$ with an rf voltage of $1 \mathrm{MV}$, and the synchronous phase $\phi_{s}=\sin ^{-1} \Gamma$ is taken to be zero. This supplies Landau damping. The mode will be stable if

$$
\frac{1}{\tau}<\frac{\sqrt{m}}{4} \Delta \omega_{s}=1.96 \sqrt{m} \mathrm{~s}^{-1}
$$

The Landau damping rates are listed in the last column of Table V, and the modes that receive not enough Landau damping are underlined.

For the $140 \times 121$ scenario, the growth rates can be obtained by linearly scaling the number of bunches $M$. Of course, the growth of all modes will be faster.

We would like to point out that the inductive impedance gives rise to an incoherent synchrotron frequency shift of

$$
\frac{\Delta \omega_{s}}{\omega_{s}}=-\frac{3 I_{b} \operatorname{Im}\left(Z_{\|} / n\right)}{2 \pi^{2} h V_{\mathrm{rf}} \cos \phi_{s} B_{0}^{3}}=-0.0463 \quad \text { or } \quad \Delta f_{s}=-4.05 \mathrm{~Hz}
$$




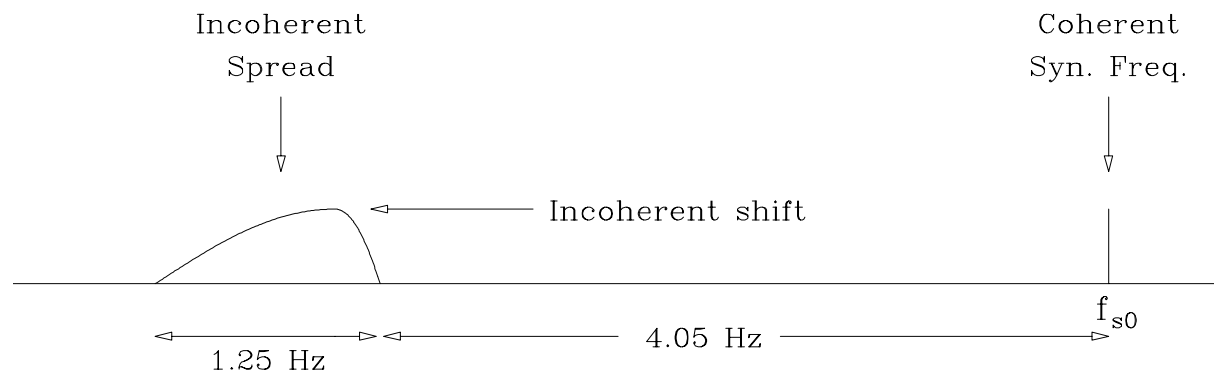

Figure 12: Schematic drawing showing the incoherent spread of $\Delta f_{s} \approx 1.25 \mathrm{~Hz}$ is shifted by $-4.05 \mathrm{~Hz}$ from the coherent synchrotron frequency $f_{s 0}$, thus not being able to provide Landau damping to the dipole $(m=1)$ modes.

where $\operatorname{Im}\left(Z_{\|} / n\right)=3 \Omega$ has been used. However, the coherent synchrotron frequency remains the same as the unperturbed synchrotron frequency $f_{s 0}$. Thus the incoherent spread of the synchrotron frequency will not cover $f_{s 0}$, and will not supply any damping to the $m=1$ mode. This is illustrated in Fig. 12 . The sizes of the incoherent frequency shift and spread depend rather sensitively on the bunch distribution. For example, for a cosine-square distribution and a Gaussian distribution with the same rms bunch length, the incoherent frequency shifts will be, respectively, $\sim 1.74$ or $\sim 2.97$ times larger than that of the parabolic distribution. Also due to the nonuniform distribution gradients in these two distributions, the incoherent frequency spreads will also be broader. Nevertheless, the conclusion is qualitatively the same. For all reasonable distributions, the incoherent frequency spread will not be able to overlap the coherent dipole synchrotron frequency, resulting in no Landau damping.

We see from Table $\mathrm{V}$ that the azimuthal mode $m=1$ driven by the resonance at $310.7 \mathrm{MHz}$ will growth at a rate of 2.33 per second. Although the growth rate is small, however, the growth is severe because the ramp rate of the Tevatron is slow; the energy reaches only $\sim 220 \mathrm{GeV}$ after ramping for $20 \mathrm{~s}$. In computing the growth rates in Table $\mathrm{V}$, we have assumed that the resonant peaks of the 8 cavities do not fall on top of each other and the effective peak of the sum broadened. We took the shunt impedance to be the shunt impedance of the resonance of one cavity and increase the quality factor 8-fold. In this way, the FWHM is 3.27 revolution harmonics and the decay decrement of the resonant field is $\alpha \tau_{\mathrm{sep}}=0.194$. From Fig. 10, it is clear that the function $D\left(\alpha \tau_{\mathrm{sep}}\right) \approx 1$. However, if we assume the resonant peaks of the 8 cavities to fall on top of each other, the situation will be different. Although the decay decrement is 0.0242 and $D$ is still equal to unity, the FWHM is only 0.409 revolution harmonic. This implies that resonant may not fall on top of an upper synchrotron side-band of a harmonic line, and if this happens the growth rate will be very much reduced. Unfortunately, the resonant frequencies measured is not accurate enough for us to decide whether they are near a revolution harmonic or not.

If the growth turns out to be harmful, a fast $36 \times 36$ bunch by bunch damper may be necessary to damp the dipole mode $(m=1)$. A damper for the quadrupole mode $(m=2)$ may also be necessary. This consists essentially of a wall-gap pickup monitoring the changes in bunch length and the corresponding excitation of a modulation of the rf waveform with roughly twice the synchrotron frequency. The Tevatron bunches 
will be formed by coalescing 9 or more bunches in the Main Injector (formerly in the Main Ring). Usually there will be a $10 \%$ difference in the number of particles in the final bunches. This difference will break the symmetry of the coupled-bunch system and lead to some damping also.

We would like also to compute the longitudinal coupled bunch growth rates for Run I, where there were only 6 proton bunches with a rms length of $85.5 \mathrm{~cm}$ and the same number of protons per bunch as in Run II. A smaller number of bunches will certainly reduce the growth rates. The longer bunch length will make the driving force less effective because of the much larger change in phase of the resonator during the passage of the bunch. Also a bigger bunch in the longitudinal phase space will provide more Landau damping. The growth rates at $150 \mathrm{GeV}$ are listed in Table VI. We see that Landau damping prevents all

Table VI: Longitudinal coupled-bunch growth rates driven by the higher-order modes of the rf cavities at injection for the $6 \times 6$ scenario in Run I.

\begin{tabular}{crrrrrrrrr}
\hline$f_{r}$ & $R_{s}$ & $Q$ & $\Delta \phi$ & \multicolumn{6}{c}{ Growth Rate in sec $^{-1}$} \\
$\mathrm{MHz}$ & $\mathrm{k} \Omega$ & & $\mathrm{rad}$ & $m=1$ & $m=2$ & $m=3$ & $m=4$ & $m=5$ & $m=6$ \\
\hline 56.5 & 68 & 3620 & 1.91 & $\underline{0.090}$ & 0.007 & 0.000 & 0.000 & 0.000 & 0.000 \\
158.2 & 70 & 6060 & 5.34 & $\underline{\underline{0.090}}$ & 0.072 & 0.019 & 0.003 & 0.000 & 0.000 \\
310.7 & 124 & 15923 & 10.48 & $\underline{\underline{0.035}}$ & 0.067 & 0.105 & 0.081 & 0.034 & 0.009 \\
424.2 & 8 & 6394 & 14.32 & $\underline{\underline{0.001}}$ & 0.003 & 0.003 & 0.005 & 0.005 & 0.003 \\
439.8 & 71 & 13728 & 14.84 & $\underline{0.010}$ & 0.022 & 0.027 & 0.037 & 0.045 & 0.032 \\
559.5 & 93 & 13928 & 18.88 & $\underline{0.009}$ & 0.016 & 0.025 & 0.032 & 0.033 & 0.043 \\
748.2 & 145 & 13356 & 25.25 & $\underline{0.008}$ & 0.014 & 0.022 & 0.028 & 0.033 & 0.042 \\
768.0 & 39 & 16191 & 25.92 & $\underline{0.002}$ & 0.004 & 0.006 & 0.008 & 0.009 & 0.011 \\
\hline Laudau & \multicolumn{1}{l}{ Damping rate $\left(\mathrm{s}^{-1}\right)$} & 0.000 & 2.626 & 3.212 & 3.709 & 4.149 & 4.546 \\
\hline
\end{tabular}

azimuthal coupled-bunch modes with $m>1$ from instabilities. The only unstable modes are the dipole modes which have no Landau damping. However, the highest growth rate is only $0.090 \mathrm{~s}^{-1}$. Such slow rate would be damped by the slight unequal number of particles in the bunches. This may explain why no longitudinal coupled-bunch instabilities had been observed during Run I.

\section{LONGITUDINAL HEAD-TAIL INSTABILITY}

In general, the slippage factor $\eta$ is not an even function of momentum offset and the particle trajectory will be asymmetric about the on-momentum axis. When the first-order coefficient $\alpha_{0} \alpha_{1}$ of momentum compaction factor is positive, the particle spends more time at positive momentum offset than at negative momentum offset. Thus the bunch becomes relatively longer at positive momentum offset than at negative momentum offset, as is illustrated in Fig. 13. The bunch will therefore lose more energy in the lower trajectory than in the upper trajectory. The amplitude of synchrotron oscillation will therefore grow. This phenomenon is called longitudinal head-tail instability and was first observed at the CERN PS by Boussard 


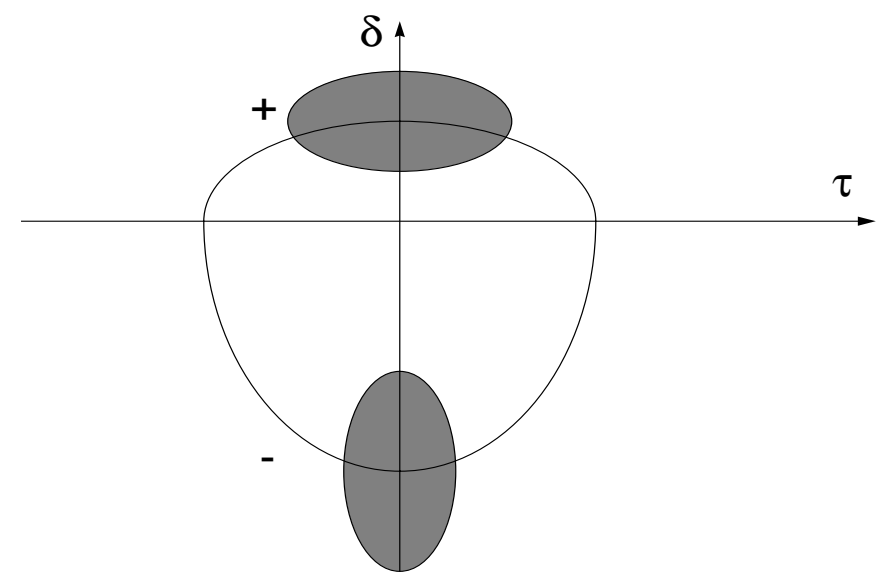

Figure 13: A particle trajectory is asymmetric about the on-momentum axis when the slippage factor is not an even function of momentum offset. The bunch will be longer at positive than negative momentum offset when the first-order momentum compaction $\alpha_{0} \alpha_{1}>0$ and above transition.

and Linnecar [13]. The growth rate is given by

$$
\frac{1}{\tau}=-\frac{f_{0}}{2} \frac{d U}{d \sigma_{\tau}} \frac{\sigma_{\tau}}{E} \chi
$$

where the energy loss per particle per turn is

$$
U\left(\sigma_{\tau}\right)=e^{2} N \int d \omega|\tilde{\rho}(\omega)|^{2} \operatorname{Re} Z_{\|}(\omega),
$$

and

$$
\chi=\frac{\alpha_{0}\left(\alpha_{1}-\eta+\frac{3}{2}\right)}{\eta} \approx \alpha_{1}+\frac{3}{2}
$$

denotes the asymmetry, which has been measured to be $\chi \sim+1.17$ for the Tevatron. In the above,

$$
\tilde{\rho}(\omega)=\frac{1}{2 \pi} \int d \tau \rho(\tau) e^{-j \omega \tau}
$$

is the spectrum of the bunch of rms length $\sigma_{\tau}$ with a distribution $\rho(\tau)$ normalized to unity.

If the driving impedance $\operatorname{Re} Z_{\|}$comes from a narrow resonance with shunt impedance $R_{s}$ at resonant frequency $\omega_{r} /(2 \pi)$ and quality factors $Q$, we have for the energy loss per turn

$$
U\left(\sigma_{\tau}\right)=\frac{\pi R_{s} \omega_{r} e^{2} N}{Q}\left|\tilde{\rho}\left(\omega_{r}\right)\right|^{2}
$$

for a bunch containing $N$ particles. For a broad-band impedance, $U\left(\sigma_{\tau}\right)$ drops much faster with bunch length. For a general resonance, we have computed the asymmetric energy loss for a parabolic bunch distribution,

$$
\frac{d U\left(\sigma_{\tau}\right)}{d \sigma_{\tau}} \sigma_{\tau}=\frac{9 e^{2} N \omega_{r} R_{s}}{4 s Q}\left\{\frac{2}{z^{3}}\left[e^{-2 c z} \sin (2 s z+2 \theta)-\sin 2 \theta\right]\right.
$$




$$
\begin{aligned}
& +\frac{4}{z^{4}}\left[e^{-2 c z} \sin (2 s z+3 \theta)+\sin 3 \theta\right]+\frac{12}{z^{5}} e^{-2 c z} \sin (2 s z+4 \theta) \\
& \left.+\frac{6}{z^{6}}\left[e^{-2 c z} \sin (2 s z+5 \theta)+\sin 5 \theta\right]\right\},
\end{aligned}
$$

where $z=\sqrt{5} \omega_{r} \sigma_{\tau}, c=\cos \theta=1 /(2 Q)$, and $s=\sin \theta$. This is plotted in Fig. 14 for the case of a sharp resonance and in Fig. 15 for the case of a broad-band with $Q=1$. As is shown in Fig. 14, the asymmetric energy loss vanishes when the bunch length goes to zero, because the change in bunch length from positive momentum offset to negative momentum offset also goes to zero. On the other hand, when the bunch length is very long, the asymmetric energy loss will also be small, because the energy loss for a long bunch is small.

The fundamental resonance of the $8 \mathrm{rf}$ cavities serves as a good driving force for this instability. Each cavity has resonant frequency $f_{r}=53.1 \mathrm{MHz}, R_{s}=1.2 \mathrm{M} \Omega$, and $Q=7000$. For Run I, where the rms bunch length was $\sigma_{\tau} \approx 2.684 \mathrm{~ns}$ or $f_{r} \sigma_{\tau} \approx 0.1425,\left(d U / d \sigma_{\tau}\right) \sigma_{\tau} \sim-0.3890 e^{2} N \omega_{r} R_{s} / Q$ is large and leads to a growth rate of $\tau^{-1}=1.433 \times 10^{-3} \mathrm{~s}^{-1}$ at the injection energy of $E=150 \mathrm{GeV}$ for a bunch containing $N=2.70 \times 10^{11}$ particles. However, for Run II, the bunch will be much shorter. With $\sigma_{\tau}=1.234 \mathrm{~ns}$ or $f_{r} \sigma_{\tau} \approx 0.0655$, the asymmetric energy loss $\left(d U / d \sigma_{\tau}\right) \sigma_{\tau} \sim-0.1464 e^{2} N \omega_{r} R_{s} / Q$ is much smaller and the head-tail growth rate becomes $\tau^{-1}=0.539 \times 10^{-3} \mathrm{~s}^{-1}$. As is shown in Fig. 14, we are on the left side of the $\left(d U / d \sigma_{\tau}\right) \sigma_{\tau}$ peak; therefore a shorter bunch length leads to slower growth.

The broad-band impedance can also have similar contributions since the resonance frequency is usually a few $\mathrm{GHz}$ and $\operatorname{Re} Z_{\|}$is large although $Z_{\|} / n$ is just a couple of ohms. Now $\omega_{r} \sigma_{\tau}$ falls on the right side of the $\left(d U / d \sigma_{\tau}\right) \sigma_{\tau}$ peak instead. We expect shorter bunch lengths to have faster growth rates, as is indicated in Fig. 15. Table VII shows the longitudinal head-tail growth rates for different resonant frequencies and

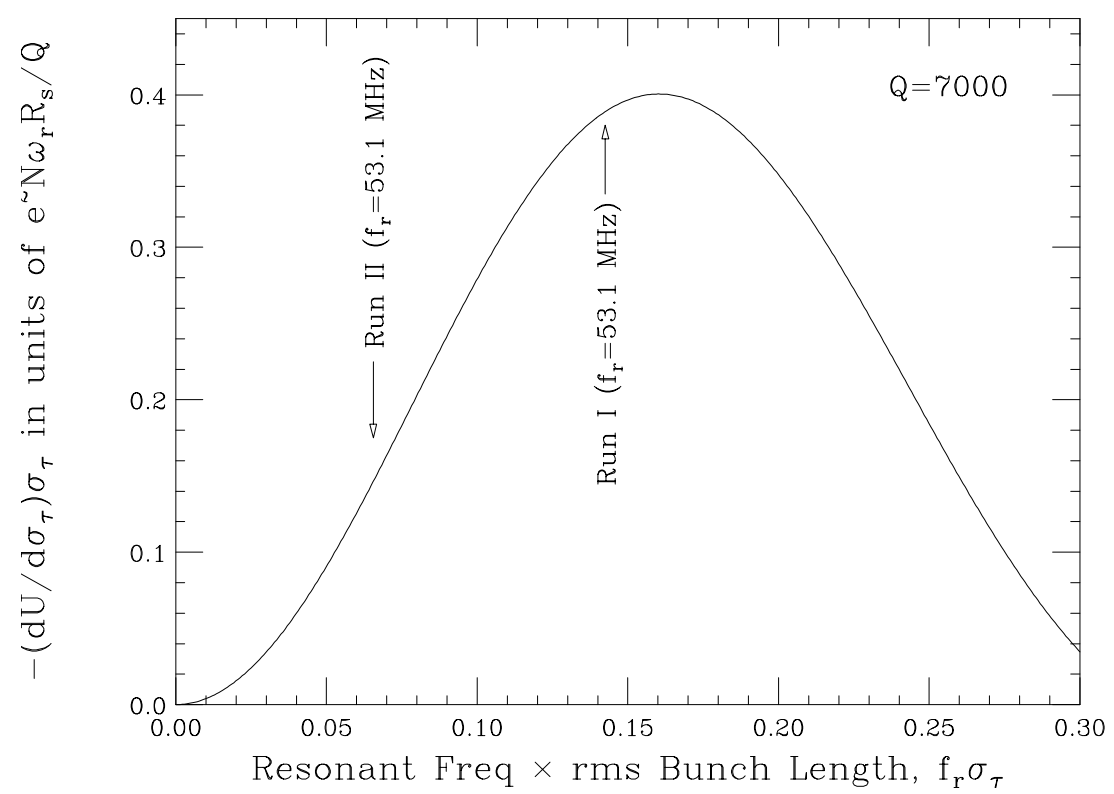

Figure 14: Plot of differential bunch energy loss $\left(d U / d \sigma_{\tau}\right) \sigma_{\tau}$ verses $f_{r} \sigma_{\tau}$ due to a sharp resonance. Note that the effect on the Run II bunch is much less than that on the Run I bunch because of the shorter Run II bunch length. 


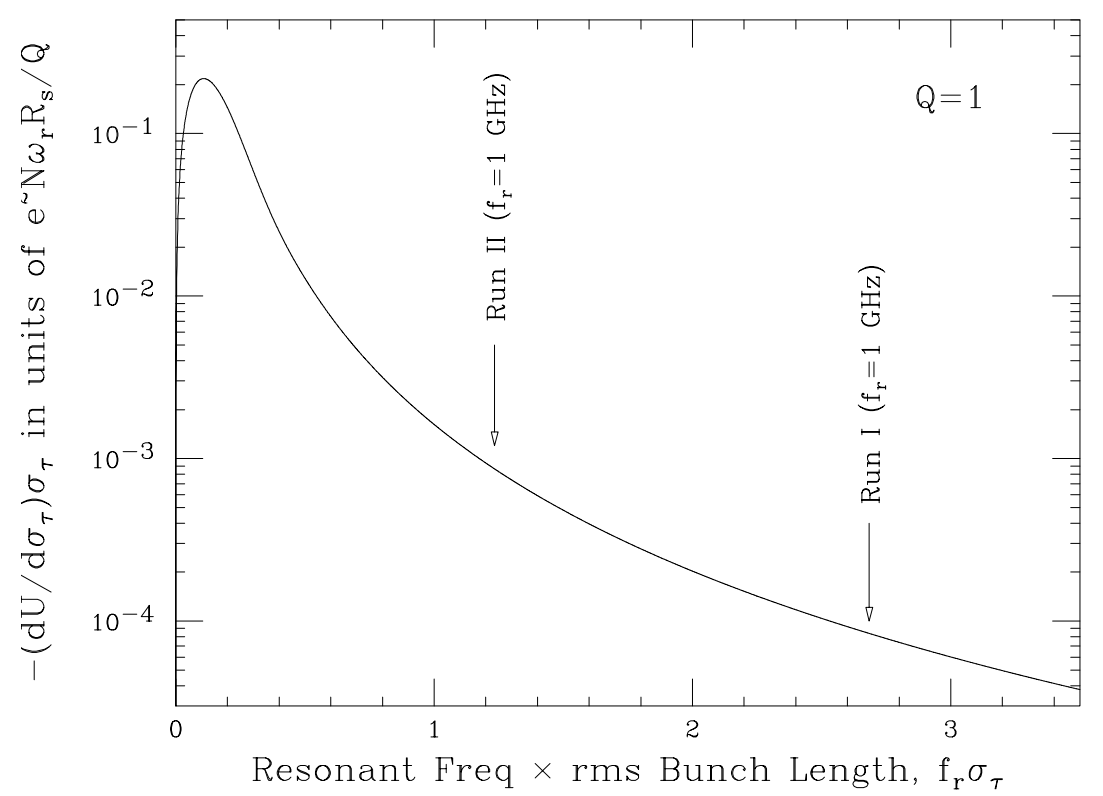

Figure 15: Plot of differential bunch energy $\operatorname{loss}\left(d U / d \sigma_{\tau}\right) \sigma_{\tau}$ verses $f_{r} \sigma_{\tau}$ due to a broad-band resonance with $Q=1$. Note that the effect on the Run II bunch is much more than that on the Run I bunch because of the shorter Run II bunch length.

quality factors; $Z_{\|} / n=2 \Omega$ has been assumed. The growth rates driven by the fundamental rf resonance are also listed in the last row for comparison. It is obvious that the longitudinal head-tail instability for Run I is dominated by the rf narrow resonance and that for Run II by the broad-band impedance instead. We observed a growth time of $\sim 250 \mathrm{~s}$ in Run I. From Table VI, it is very plausible that the growth of this head-tail instability will be at least as fast as that in Run I.

Table VII: Growth rates for a broad-band resonance of $Z_{\|} / n=2 \Omega$ at various frequencies and quality factors.

\begin{tabular}{cccc}
\hline$f_{r}(\mathrm{GHz})$ & $Q$ & \multicolumn{2}{c}{ Growth Rate $\left(\mathrm{s}^{-1}\right)$} \\
& & Run I & Run II \\
\hline 1 & 1 & $0.178 \times 10^{-3}$ & $1.829 \times 10^{-3}$ \\
1 & 3 & $0.022 \times 10^{-3}$ & $0.267 \times 10^{-3}$ \\
2 & 1 & $0.089 \times 10^{-3}$ & $0.915 \times 10^{-3}$ \\
2 & 2 & $0.023 \times 10^{-3}$ & $0.249 \times 10^{-3}$ \\
1 & 5 & $0.009 \times 10^{-3}$ & $0.114 \times 10^{-3}$ \\
2 & 3 & $0.011 \times 10^{-3}$ & $0.117 \times 10^{-3}$ \\
2 & 4 & $0.006 \times 10^{-3}$ & $0.070 \times 10^{-3}$ \\
\hline \multicolumn{2}{l}{ Fundamental Rf Resonance } & $1.433 \times 10^{-3}$ & $0.539 \times 10^{-3}$ \\
\hline
\end{tabular}




\section{TRANSVERSE MICROWAVE INSTABILITY}

Similar to the longitudinal case, the beam current at a certain betatron spectral frequency $\left(n_{r}+\nu_{\beta}\right) f_{0}$ interacts with the transverse impedance to create a transverse deflecting force leading to an enhancement of the amplitude of the betatron oscillation. Here, $n_{r}$ is a revolution harmonic and $\nu_{\beta}$ is the betatron tune. We need to consider only the slow wave that can cause instability and therefore $n_{r}<0$. This growth can be damped by the incoherent spread of the betatron spectral line under consideration. As a result of momentum spread $\delta$, this incoherent spread is

$$
\Delta f_{\beta}=\left[-\left(n_{r}+\nu_{\beta 0}\right) \eta+\xi\right] f_{0} \delta,
$$

where $\nu_{\beta 0}$ is the on-momentum betatron tune and $\xi$ the chromaticity. Applying to a bunch, we can therefore write down a Keil-Schnell type of stability criterion [14]:

$$
\left|Z_{\perp}\right|<\frac{4 \sqrt{2} E \nu_{\beta 0}}{e I_{\mathrm{pk}} R}\left|\left(\left|n_{r}\right|-\nu_{\beta 0}\right) \eta+\xi\right| \delta_{\mathrm{FWHM}}
$$

where $R$ is the mean radius of the accelerator ring. Similar to our discussion in Sec. IV, if the momentum spread is inferred from Eq. (3.4), it will be diminished by the square of the bunch lengthening factor $k$ as a result of the inductive impedance.

Since the bunch length is much larger than the beam pipe radius, the half-wavelength of the driving impedance force will be less than the full length of the bunch. We therefore take the perturbing frequency as $f_{r}=1 /(4 \hat{\tau})=90.6 \mathrm{MHz}$ or $\left|n_{r}\right|=1 /\left(4 \hat{\tau} f_{0}\right)=1899$, and obtain the stability limit $\left|Z_{\perp}\right|<3.26 \mathrm{M} \Omega / \mathrm{m}$ at zero chromaticity and injection energy. Note the $\left|Z_{\perp}\right|$ near this frequency is 3 to $4 \mathrm{M} \Omega / \mathrm{m}$ from Fig. 9 together with other discontinuities of the vacuum chamber. Thus, transverse microwave instability will be plausible in Run II. However, a chromaticity of $\xi=+10$ implies raising $\left|n_{r}\right|$ effectively by $\xi / \eta=3537$ and increasing the stability limit to $\left|Z_{\perp}\right|<9.41 \mathrm{M} \Omega / \mathrm{m}$. On the other hand, a negative chromaticity will lower the stability limit and lead to instability.

\section{TRANSVERSE COUPLED-BUNCH INSTABILITIES}

\section{VIII.1 RESISTIVE WALL}

A most serious transverse coupled-bunch instability in a storage ring may be driven by the resistive wall. If there are $M_{s}$ identical equally spaced bunches in the ring, there are $\mu=0, \cdots, M_{s}-1$ transverse coupled modes when the centers of mass of one bunch lags behind its predecessor by the betatron phase of $2 \pi \mu / M_{s}$. At the same time, each bunch can execute longitudinal motion with $m=0,1, \cdots$ nodes. The growth rate for the mode $\mu m$ is [15]

$$
\frac{1}{\tau_{\mu m}}=-\frac{1}{1+m} \frac{e M I_{b} c}{4 \pi \nu_{\beta} E} \sum_{k} \operatorname{Re} Z_{\perp}\left[\left(k M_{s}-\mu+\nu_{\beta}+m \nu_{s}\right) \omega_{0}\right] F_{m}^{\prime}\left(\omega \tau_{L}-\chi\right)
$$

where $M$ is the number of bunches. Strictly speaking Eq. (8.1) is correct only if $M=M_{s}$ or a completely filled ring. For example, in the $36 \times 36$ scenario, the bunch spacing is 21 buckets; therefore $M=36$ and 


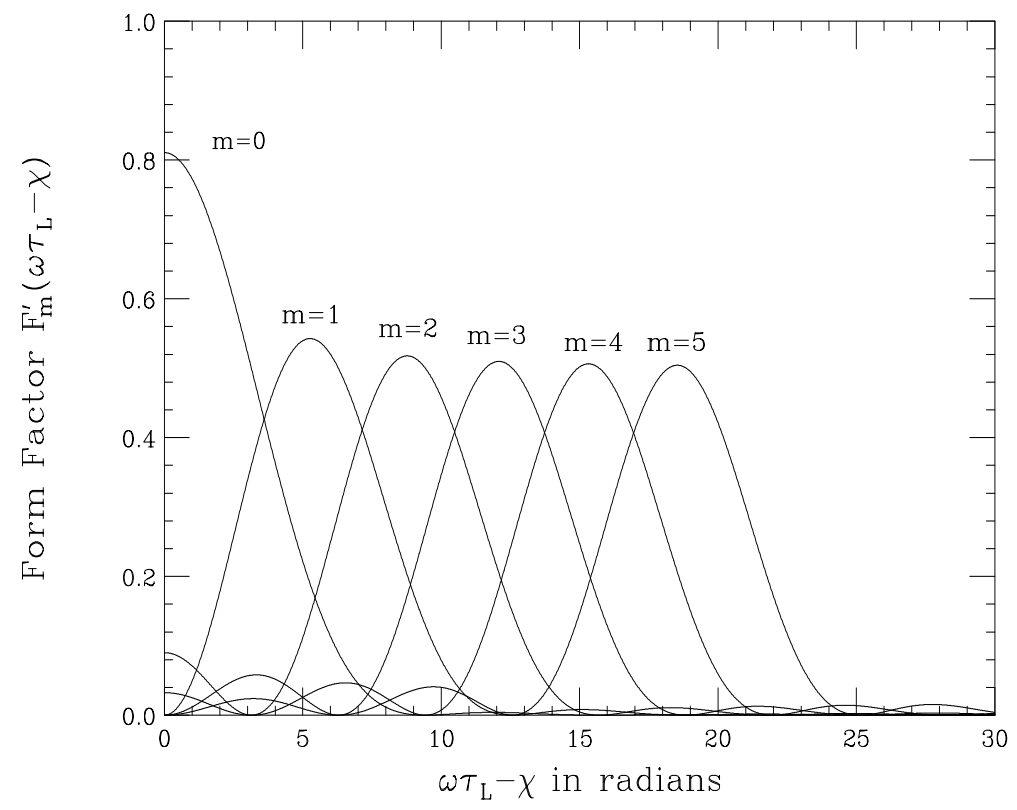

Figure 16: Plot of form factor $F_{m}^{\prime}\left(\omega \tau_{L}-\chi\right)$ for modes $m=0$ to 5 . With the normalization in Eq. (8.3), these are exactly the power spectra $h_{m}$.

$M_{s}=1113 / 21=53$, and in the $140 \times 121$ scenario, $M=140$ (for protons) and $M_{s}=159$. There are many unfilled buckets in both scenarios; thus Eq. (8.1) will not be an accurate description of the beam dynamics.

As the frequency $\omega \rightarrow \pm 0$, the real part of the resistive-wall impedance approaches first $\pm|\omega|^{-1 / 2}$, then $|\omega|^{-1}$ when the skin depth exceeds the thickness of the pipe wall, and finally zero when the frequency is exactly zero. At the residual betatron tune of the Tevatron, $\left[\nu_{\beta}\right] \sim \pm 0.4$, we are in the regime of $\pm|\omega|^{-1 / 2}$ dependency. Therefore, there is always a mode $\mu$ that corresponds to a large negative $\operatorname{Re} Z_{\perp}$ and drives the transverse coupled-bunch instability. For example, with the betatron tune $\nu_{\beta}=20.57$, mode $\mu=21$ or frequency $-0.43 \omega_{0} /(2 \pi)$ with $k=0$ in the summation of Eq. (8.1) contributes the largest negative $\operatorname{Re} Z_{\perp}$, which is $-66.70 \mathrm{M} \Omega / \mathrm{m}$ according to our former estimate made in Sec. II.2. The next contribution with $k=1$ will give $\operatorname{Re} Z_{\perp}=+6.03 \mathrm{M} \Omega / \mathrm{m}$ in the $36 \times 36$ scenario and $+3.47 \mathrm{M} \Omega / \mathrm{m}$ for protons in the $140 \times 121$ scenario. The average current per bunch is $I_{b}=2.064 \mathrm{~mA}$. The growth rate is therefore given mostly by the $k=0$ term in the summation and is very insensitive to the choice of $M_{s}$ in Eq. (8.1). For such a low driving frequency, only the lowest longitudinal mode $m=0$ will be excited. The growth rates after doing the actual summations are 31.0 and $120.6 \mathrm{~s}^{-1}$, respectively, for the two scenarios. Modes $\mu=22,23,24, \cdots$ are also unstable; the growth rates are, respectively, $16.9,12.8,10.6, \cdots \mathrm{s}^{-1}$, and $66.1,50.6,42.5, \cdots \mathrm{s}^{-1}$ for the two operating scenarios. The computation has been performed at zero chromaticity $(\xi=0)$, so that the chromatic phase $\chi=\xi \omega_{0} \tau_{L} / \eta=0$. Also, we have used the form factor $F_{0}^{\prime}(0)=8 / \pi^{2} \approx 0.811$, where, for convenience, Sacherer's sinusoidal modes of excitation have been assumed. These growth rates are much larger than those in Run I because there are more bunches. If one operates at chromaticity $\xi=+10, \chi=5.85$, $F_{0}^{\prime}(5.85) \approx 0.155$ from Fig. 16 . The growth rates for $\mu=21$ drop to 5.9 and $9.7 \mathrm{~s}^{-1}$, respectively, which can be damped easily by a tune spread. For example, a tune spread of $\Delta \nu_{\beta}=0.0001$ will lead to a spread of 
betatron angular frequency of $\Delta \nu_{\beta} \omega_{0}=30 \mathrm{~s}^{-1}$, and will damp a growth rate up to $\sim 17.0 \mathrm{~s}^{-1}$ (FWHM for a Gaussian spread) [15]. For further discussion, we need to study the sinusoidal modes of excitation in the next subsection.

\section{VIII.2 SINUSOIDAL MODES}

The Sacherer's sinusoidal modes of excitation consist of the orthonormal set

$$
p_{m}(\tau)=\left\{\begin{array}{cc}
\cos (m+1) \pi \frac{\tau}{\tau_{L}} & m=0,2, \cdots, \\
\sin (m+1) \pi \frac{\tau}{\tau_{L}} & m=1,3, \cdots
\end{array}\right.
$$

such that $p_{m}(\tau)$ has $m$ nodes along the bunch not including the ends. The power spectrum is proportional to

$$
h_{m}(\omega)=\frac{4(m+1)^{2}}{\pi^{2}} \frac{1+(-1)^{m} \cos \pi y}{\left[y^{2}-(m+1)^{2}\right]^{2}}
$$

where $y=\omega \tau_{L} / \pi$ and $\omega=k M-\mu+\nu_{\beta}+m \nu_{s}-\chi / \tau_{L}$. They are plotted in Fig. 17. The normalization of $h_{m}(\omega)$ in Eq. (8.3) has been chosen in such a way that, when the smooth approximation is applied to the summation over $k$, we have

$$
B \sum_{k=-\infty}^{+\infty} h_{m}(\omega) \approx \frac{B}{M \omega_{0}} \int_{-\infty}^{+\infty} h_{m}(\omega) d \omega=1
$$

Here $B=M \omega_{0} \tau_{L} /(2 \pi)$ is the bunching factor, or the ratio of full bunch length to bunch separation. Then the form factor $F_{m}^{\prime}(\omega)$ in Eq. (8.1) just equals $h_{m}(\omega)$.

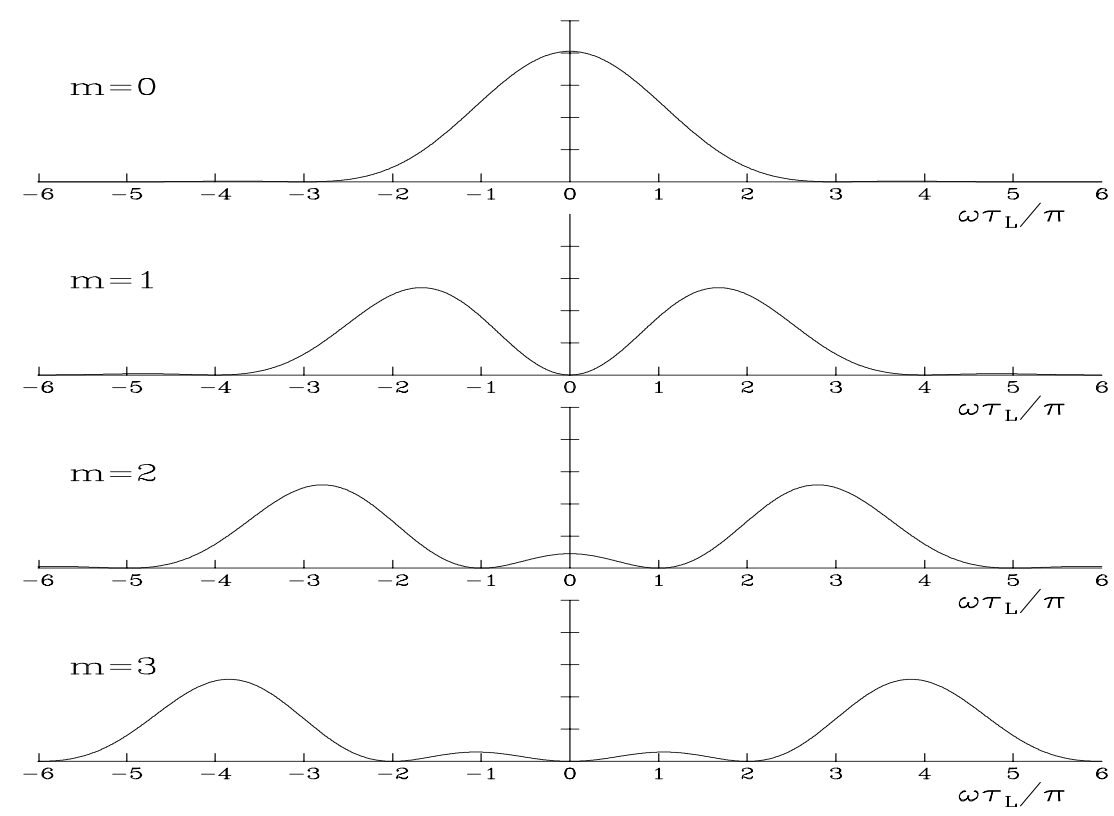

Figure 17: Power spectra $h_{m}(\omega)$ for modes $m=0$ to 3 with zero chromaticity. 
The Sacherer integral equation for transverse instability is an eigen-value-eigen-function problem when the unperturbed longitudinal distribution $g_{0}(r)$ in the longitudinal phase space is given. Physically, the modes of excitation $p_{m}(\tau)$ are the projection of the eigen-functions in the longitudinal phase space onto the time axis. The sinusoidal modes corresponds to the water-bag distribution ${ }^{\dagger}$

in phase space, so that the linear distribution is

$$
\rho(\tau) \propto \sqrt{\hat{\tau}^{2}-\tau^{2}}
$$

For the distribution $g_{0}(r) \propto\left(\hat{\tau}^{2}-r^{2}\right)^{-1 / 2}$ in the longitudinal phase space, $p_{m}(\tau)$ are the Legendre polynomials and the Fourier transforms the spherical Bessel functions $j_{m}$. When $g_{0}(r)$ is bi-Gaussian, $p_{m}(\tau)$ are Hermite polynomials. Sometimes the growth rates computed are rather sensitive to the longitudinal bunch distribution assumed. Therefore, results in this section are estimates only.

We now learn that a chromaticity of $\xi=\eta /\left(f_{0} \tau_{L}\right)=+10.73$ will push the power spectra in Fig. 17 to the right (or positive frequency side) by two $\omega \tau_{L} / \pi$ units. The $m=0$ will then only see the positive-frequency impedance and no instability will result. However, the $m=1$ mode will now peak at zero frequency and the resistive wall impedance will drive the $m=1$ mode unstable and a quadrupole transverse damper will be required.

\section{VIII.3 TRANSVERSE COUPLED-BUNCH INSTABILITY DRIVEN BY RESONANCES}

The narrow transverse resonant modes of the rf cavities will also drive transverse coupled-bunch instability. The growth rate is described by the general growth formula of Eq. (8.1). When the resonance is narrow enough, only one frequency $-\omega_{r} /(2 \pi)$ contributes in the summation. Thus the growth rate becomes

$$
\frac{1}{\tau_{\mu m}}=-\frac{1}{1+m} \frac{e M I_{b} c}{4 \pi \nu_{\beta} E} \mathcal{R e} Z_{\perp}\left(\omega_{r}\right) F_{m}^{\prime}\left(\omega_{r} \tau_{L}-\chi\right),
$$

where $\omega_{r}$ is negative. We calculated the growth rates of modes driven by the nine higher-order dipole modes computed by URMEL in Table III. The results are listed in Table VIII.

Some comments are in order. Here, we assume that the higher-order modes of the $8 \mathrm{rf}$ cavities do not fall on top of each other at exactly the same frequency. In other words, we assume the resonances summed over 8 cavities will be de-Qued 8 times and the shunt impedance corresponding to a certain mode will be the same as that for a single cavity. From Table VIII, we see that the frequencies of the lowest 9 higher-order modes range from 486.5 to $699.7 \mathrm{MHz}$. Therefore $\omega_{r} \tau_{L} / \pi-\chi / \pi\left(\omega_{r}\right.$ is negative) ranges from 5.4 to 7.4 for zero chromaticity. From the power spectra in Fig. 17, this implies negative resonant frequencies $\omega_{r}$ are exciting the modes that peak in the region, or modes roughly from $m=4$ to 7 . These are listed in column 4 of the table. We can see, for example, that the growth rate driven by the first resonance at zero chromaticity

\footnotetext{
${ }^{\dagger}$ In the Sacherer integral equation for transverse instability, the weight function is $W(r)=g_{0}(r)$. However, in the integral equation for longitudinal instability, the weight function is $W(r)=-r^{-1} g_{0}^{\prime}(r)$. As a result, for that equation, the sinusoidal modes correspond roughly to $g_{0}(r) \propto\left(\hat{\tau}^{2}-r^{2}\right)$, the Legendre modes correspond to $g_{0}(r) \propto\left(\hat{\tau}^{2}-r^{2}\right)^{1 / 2}$. But the Hermite modes correspond to the same bi-Gaussian distribution.
} 
Table VIII: Growth rates for transverse coupled-bunch modes driven by higher-order dipole modes of the rf cavities.

\begin{tabular}{|c|c|c|c|c|c|c|c|c|c|c|}
\hline \multirow{2}{*}{$\begin{array}{c}f_{r} \\
\mathrm{MHz}\end{array}$} & \multirow{2}{*}{$\begin{array}{c}R_{s} \\
\Omega / m\end{array}$} & \multirow[t]{2}{*}{$Q$} & \multirow[t]{2}{*}{$m_{\mathrm{pk}}$} & \multicolumn{7}{|c|}{ Growth Rate $\left(\mathrm{s}^{-1}\right)$} \\
\hline & & & & Growth & $m=0$ & $m=1$ & $m=2$ & $m=3$ & $m=4$ & $m=5$ \\
\hline \multicolumn{11}{|c|}{ Chromaticity $\xi=0$} \\
\hline 486.5 & 7262 & 31605 & 4.4 & 4.173 & 0.001 & 0.008 & 0.008 & 0.057 & 0.346 & 0.276 \\
\hline 486.9 & 4689 & 31487 & 4.4 & 2.694 & 0.001 & 0.005 & 0.005 & 0.036 & 0.223 & 0.179 \\
\hline 513.4 & 3904 & 33262 & 4.7 & 2.243 & 0.001 & 0.001 & 0.008 & 0.007 & 0.135 & 0.180 \\
\hline 518.3 & 4010 & 34008 & 4.7 & 2.304 & 0.002 & 0.001 & 0.008 & 0.005 & 0.128 & 0.189 \\
\hline 561.7 & 2695 & 33029 & 5.2 & 1.549 & 0.001 & 0.000 & 0.004 & 0.001 & 0.031 & 0.121 \\
\hline 575.3 & 137 & 35810 & 5.4 & 0.079 & 0.000 & 0.000 & 0.000 & 0.000 & 0.001 & 0.006 \\
\hline 625.1 & 1988 & 32598 & 5.9 & 1.142 & 0.000 & 0.001 & 0.000 & 0.004 & 0.000 & 0.040 \\
\hline 650.9 & 1323 & 37592 & 6.2 & 0.760 & 0.000 & 0.000 & 0.000 & 0.002 & 0.000 & 0.014 \\
\hline 699.7 & 1829 & 33407 & 6.7 & 1.051 & 0.000 & 0.000 & 0.001 & 0.000 & 0.003 & 0.002 \\
\hline \multicolumn{11}{|c|}{ Chromaticity $\xi=+10$} \\
\hline 486.5 & 7262 & 31605 & 6.2 & 4.173 & 0.000 & 0.003 & 0.001 & 0.009 & 0.003 & 0.066 \\
\hline 486.9 & 4689 & 31487 & 6.2 & 2.694 & 0.000 & 0.002 & 0.000 & 0.006 & 0.002 & 0.042 \\
\hline 513.4 & 3904 & 33262 & 6.5 & 2.243 & 0.000 & 0.001 & 0.001 & 0.002 & 0.005 & 0.012 \\
\hline 518.3 & 4010 & 34008 & 6.6 & 2.304 & 0.000 & 0.000 & 0.002 & 0.002 & 0.006 & 0.009 \\
\hline 561.7 & 2695 & 33029 & 7.1 & 1.549 & 0.000 & 0.000 & 0.001 & 0.000 & 0.004 & 0.000 \\
\hline 575.3 & 137 & 35810 & 7.2 & 0.079 & 0.000 & 0.000 & 0.000 & 0.000 & 0.000 & 0.000 \\
\hline 625.1 & 1988 & 32598 & 7.8 & 1.142 & 0.000 & 0.000 & 0.000 & 0.001 & 0.000 & 0.003 \\
\hline 650.9 & 1323 & 37592 & 8.0 & 0.760 & 0.000 & 0.000 & 0.000 & 0.001 & 0.000 & 0.002 \\
\hline 699.7 & 1829 & 33407 & 8.6 & 1.051 & 0.000 & 0.000 & 0.000 & 0.000 & 0.001 & 0.001 \\
\hline \multicolumn{11}{|c|}{ Chromaticity $\xi=-10$} \\
\hline 486.5 & 7262 & 31605 & 2.5 & 4.173 & 0.014 & 0.048 & 0.476 & 0.483 & 0.054 & 0.018 \\
\hline 486.9 & 4689 & 31487 & 2.5 & 2.694 & 0.009 & 0.030 & 0.306 & 0.313 & 0.035 & 0.011 \\
\hline 513.4 & 3904 & 33262 & 2.8 & 2.243 & 0.009 & 0.003 & 0.166 & 0.285 & 0.074 & 0.002 \\
\hline 518.3 & 4010 & 34008 & 2.9 & 2.304 & 0.009 & 0.002 & 0.154 & 0.294 & 0.087 & 0.001 \\
\hline 561.7 & 2695 & 33029 & 3.3 & 1.549 & 0.003 & 0.003 & 0.029 & 0.162 & 0.122 & 0.007 \\
\hline 575.3 & 137 & 35810 & 3.5 & 0.079 & 0.000 & 0.000 & 0.001 & 0.007 & 0.007 & 0.001 \\
\hline 625.1 & 1988 & 32598 & 4.0 & 1.142 & 0.000 & 0.004 & 0.000 & 0.042 & 0.113 & 0.049 \\
\hline 650.9 & 1323 & 37592 & 4.3 & 0.760 & 0.000 & 0.002 & 0.001 & 0.012 & 0.066 & 0.048 \\
\hline 699.7 & 1829 & 33407 & 4.9 & 1.051 & 0.001 & 0.000 & 0.004 & 0.000 & 0.046 & 0.088 \\
\hline
\end{tabular}


actually peaks at $m=4$. Since the growth rates are affected so much by the mode of excitation, we also give the bare growth rate for each resonance in column 5 when the form factor $F_{m}^{\prime}$ and the factor $(1+m)^{-1}$ are not included. We see that increasing the chromaticity to $\xi=+10$ shifts the mode spectra to the right (positive frequency side); so only modes of much higher $m$ will be excited. On the other hand, decreasing the chromaticity to $\xi=-10$ shifts the mode spectra to the right and lower $m$ modes will be excited. As a whole, the growth rates are slow. Since a tune spread of $\Delta \nu_{\beta}=0.0001$, for example, will damp a growth

rate up to $\sim 17 \mathrm{~s}^{-1}$. Therefore, transverse coupled-bunch instabilities driven by the higher-order modes of the rf cavities should not be a problem at all.

\section{TRANSVERSE HEAD-TAIL INSTABILITY}

Let us now consider the short-range field of the transverse impedance; i.e., $Z_{\perp}(\omega)$ when $\omega$ is large. This is equivalent to replacing the discrete line spectrum by a continuous spectrum. Since $\operatorname{Re} Z_{\perp}(\omega)$ is antisymmetric, the summation in Eq. (8.1) when transformed into an integration will vanish identically at zero chromaticity. There can only be instability when the chromaticity is nonzero.

Since the transverse impedance appears to be dominated by the resistive wall, the growth rate can be computed exactly if we substitute the impedance in Eq. (8.1) by the resistive wall formula. The result of integration is [15]

$$
\frac{1}{\tau_{m}}=-\frac{1}{1+m} \frac{e M I_{b} c}{4 \pi \nu_{\beta} E}\left(\frac{\pi}{M B}\right)^{1 / 2}\left|Z_{\perp}\left(\omega_{0}\right)\right| F_{m}(\chi),
$$

where $\left|Z_{\perp}\left(\omega_{0}\right)\right|$ is the magnitude of the resistive wall impedance at the revolution frequency. Note that the bunching factor contains a factor of $M$, so that the growth rate is actually independent of the number of bunches. This is to be expected because the growth mechanism is driven by the short-range wake field and the instability is therefore a single-bunch effect. This explains why the growth rate $\tau_{m}^{-1}$ does not contain the the subscript $\mu$ describing phase relationship of consecutive bunches.

The form factor is given by

$$
F_{m}(\chi)=\sqrt{\frac{2}{\pi}} \int_{0}^{\infty} \frac{d y}{\sqrt{y}}\left[h_{m}\left(y-y_{\xi}\right)-h_{m}\left(y+y_{\xi}\right)\right],
$$

where $h_{m}$ are the power spectra of mode $m$ in Eq. (8.3) written as functions of $y=\omega \tau_{L} / \pi$ and $y_{\xi}=\chi / \pi=$ $\xi \omega_{0} \tau_{L} /(\pi \eta)$. The first term in the integrand comes from contributions by positive frequencies while the second term by negative frequencies. The form factors for $m=0$ to 5 are plotted in Fig. 18 .

For small chromaticity $\xi \lesssim 4, \chi \lesssim 2.3$ the integrand in Eq. (9.2) can be expanded and the growth rate becomes proportional to chromaticity. From the transverse resistive wall impedance in Eq. (2.12), we obtain $\left|Z_{\perp}\left(\omega_{0}\right)\right|=61.85 \mathrm{M} \Omega / \mathrm{m}$. The growth rates for various modes have been computed and listed in Table IX, where negative growth rate implies damping rate. We see from Table VIII that mode $m=0$ is stable for positive chromaticity. This is expected because the excitation spectrum for this mode has been pushed towards the positive-frequency side. All other modes $m>0$ should be unstable because their spectra see relatively more negative $\operatorname{Re} Z_{\perp}$. However, the growth rate for $m=4$ is tiny and mode $m=2$ is even stable. 


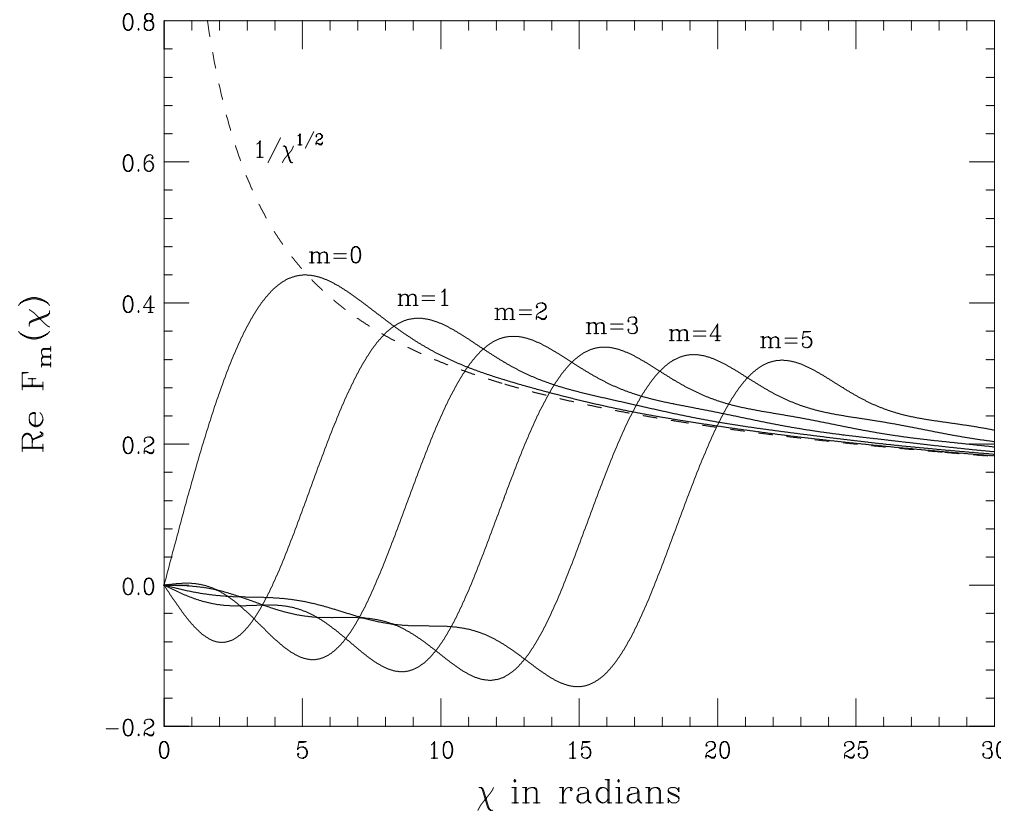

Figure 18: Form factor $F_{m}(\chi)$ for head-tail instability for modes $m=0$ to 5 .

This can be clarified by looking closely into the excitation spectra in Fig. 18. We find that while mode $m=0$ has a large maximum at zero frequency, all the other higher even $m$ modes also have small maxima at zero frequency. As these even $m$ spectra are pushed to the right, these small central maxima see more impedance from positive frequency then negative frequency. Since these small central maxima are near zero frequency where $\left|\operatorname{Re} Z_{\perp}\right|$ is large, their effect may cancel out the opposite effect from the larger maxima which interact with the impedance at much larger frequency where $\left|\mathcal{R} e Z_{\perp}\right|$ is smaller. This anomalous effect does not exist in some other longitudinal bunch distributions like $g_{0}(r) \propto\left(\hat{\tau}^{2}-r^{2}\right)^{-1 / 2}$, because the corresponding power spectra are $\left|j_{m}(\omega)\right|^{2}$ (spherical Bessel function) which vanish at zero frequency when $m>0$.

Table IX: Growth rates of transverse head-tail modes driven by the resistive wall impedance when $\chi \lesssim 2.3$.

\begin{tabular}{crr}
\hline $\begin{array}{c}\text { Mode } \\
m\end{array}$ & Form Factor & $\begin{array}{r}\text { Growth Rate } \\
\mathrm{s}^{-1}\end{array}$ \\
\hline 0 & $-0.1495 \chi$ & $-9.433 \xi$ \\
1 & $+0.0600 \chi$ & $1.893 \xi$ \\
2 & $-0.0053 \chi$ & $-0.113 \xi$ \\
3 & $+0.0191 \chi$ & $0.301 \xi$ \\
4 & $+0.0003 \chi$ & $0.003 \xi$ \\
5 & $+0.0098 \chi$ & $0.103 \xi$ \\
\hline
\end{tabular}


The head-tail instabilities can be damped by the incoherent spread in betatron frequency. As mentioned in Sec. VIII.3, a tune spread of $\Delta \nu_{\beta}=0.0001$ is capable to damp a growth rate of $\sim 17.0 \mathrm{~s}^{-1}$. Therefore, the monopole mode may not be damped if the storage ring runs at a few units of negative chromaticity. Running at positive chromaticity, however, the $m=0$ mode is stable, while the growth rates of other $m>0$ modes are small, if unstable. As an example, at $\xi=+10$ or $\chi \approx 6$, the linear approximation will no longer valid. From Fig. 18, we see that both modes $m=0$ and $m=1$ are stable. With $F_{1} \sim-0.1$, the growth rate for the $m=1$ mode is only $\sim 3.4 \mathrm{~s}^{-1}$ which will be damped by a small tune spread easily.

\section{FAST TRANSVERSE DAMPER}

A fast bunch-by-bunch transverse damper may be required to damp the bunches that have injection errors and also to cope with the possible transverse coupled-bunch instabilities.

The most important properties of a bunch-by-bunch damper are fast rise time, low pulse voltage, and low peak power. In order that the damper can kick bunch by bunch, it must be capable to recycle kick pulses in a time shorter than the bunch separation, or $132 \mathrm{~ns}$ in the $140 \times 121$ scenario, implying a full rise time less than $66 \mathrm{~ns}$. In other word, it must have a band-width broader than $7.58 \mathrm{MHz}$. With regard to the recycling time between kick pusles, the filling time of the damper pulse into the damper current plates should also be taken into consideration. The switching on and off of the damper is usually performed via a thyratron which can handle a voltage of up to roughly $60 \sim 100 \mathrm{kV}$. This sets a limit to the damper pulse voltage. At the same time, a high power source is required to generate the required damper pulses to kick the beam. For this reason, a damper design with low peak power is desired. As it turns out below, however, lowering the peak power can sometimes lead to the undesired effect of lengthening the filling time.

We are going to estimate first the power required by a damper for correcting injection offsets. The damper is placed in a location where the vertical betatron function is $\beta_{y}=100 \mathrm{~m}$. Let the maximum transverse beam offset there be $\Delta y=0.5 \mathrm{~mm}$ and we want to do the correction in $n=50$ turns. Then the angle of vertical deflection per turn by the kicker has to be

$$
\Delta y^{\prime}=\frac{\Delta y}{\beta_{y} n}=1 \times 10^{-7} .
$$

Let us consider a vertical magnetic kicker, which consists of two horizontal current plates inside a rectangular core of ferrite. These current plates are of length $\ell$ and width $w$ separated by a vertical distance $h$. Each plate is connected at one end to a 50-Ohm RG58 coaxial cable. A positive current pulse is sent into one current plate through the coaxial cable from one current source and a negative current pulse into the other from another current source so that a horizontal magnetic flux is set up between the current plates and the beam particles are kicked vertically. The other end of each current plate is terminated by a $Z_{s}=50 \Omega$ resistor hoping that the current pulse injected into each current plate will be absorbed without any reflection. The beam current will also induce electromotive (emf) forces on the plates. These emf voltages will be traveling in both directions and will also be absorbed without reflection.

Because of the high relative magnetic permeability of the ferrite, the magnetic field $\mathcal{H}$ is nearly zero 
inside the ferrite. Then between the current plates, the magnetic field $\mathcal{H}$ and magnetic flux density $\mathcal{B}$ are given by

$$
\mathcal{B}=\mu_{0} \mathcal{H}=\frac{Z_{0} I}{c w}
$$

The inductance of one current plate is therefore

$$
L=\frac{Z_{0} \ell h}{2 c w} .
$$

Putting in $w=h=6 \mathrm{~cm}$, the inductance per unit length is $L / \ell=Z_{0} h /(2 c w)=0.628 \mu \mathrm{H} / \mathrm{m}$, which is larger than the inductance $\mathcal{L}=Z_{c} / c=0.167 \mu \mathrm{H} / \mathrm{m}$ of the RG58 cable. Here, we assume that the medium inside the cable has relative dielectric $\epsilon_{r}=1$ and permeability $\mu_{r}=1$. We would like each current plate with respect to the kicker shell to behave like a transmission line matched to the RG58 cable so that currents and voltages can flow from the cable to the current plate and then the termination resistor without reflection. Therefore capacitors must be added between the current plate and the kicker shell so that the characteristic impedance equal to $Z_{c}$. The total capacitor per unit length required is

$$
\frac{C}{\ell}=\frac{L}{Z_{c}^{2} \ell}=\frac{h Z_{0}}{2 c w Z_{0}^{2}}=2.51 \mathrm{nF} .
$$

However, the phase velocity will become

$$
\frac{v_{p}}{c}=\frac{\ell}{c \sqrt{L C}}=\frac{\ell Z_{c}}{c L}=\frac{2 w Z_{c}}{h Z_{0}}
$$

We obtain $v_{p} / c \sim 0.265$ when $h \sim w$. Assuming dispersion is not important, the group velocity of the current pulse will also be $v_{g} / c \sim 0.265$.

The magnetic flux density $\mathcal{B}$ required to produce the required vertical beam deflection $\Delta y^{\prime}$ in Eq. (10.1) is given by

$$
\mathcal{B}=\frac{\beta E \Delta y^{\prime}}{e c \ell}
$$

From Eqs. (10.1) and (10.2), the minimum current in the current plates is

$$
I=\frac{\beta E \Delta y^{\prime} w}{e Z_{0} \ell} \approx 2.39 \mathrm{~A}
$$

The power dissipated is therefore

$$
P=2 I^{2} Z_{c}=571 \mathrm{~W},
$$

where the factor 2 stands for 2 circuit systems, one for each current plate.

The reduction of transmission velocity from the coaxial cable to the current plate does have some bearing on the current pulse from the generator. For example, if we need a current pulse of $I=2.39 \mathrm{~A}$ of length $\ell=1 \mathrm{~m}$ in the kicker current plate, the current sent out from the generator into the cable must be reduced to $I v_{g} / c=0.634 \mathrm{~A}$ and the length of the pulse increased to $\ell c / v_{g}=3.77 \mathrm{~m}$. The time for the pulse to fill the 1-meter kicker is $\ell / v_{g}=12.6 \mathrm{~ns}$ which is still small compared with the bunch spacing of $132 \mathrm{~ns}$.

Since each current plate behaves like a transmission line, the positive current pulse will result also in a positive voltage pulse $V=I Z_{c}$. An electric field of $\mathcal{E}=2 I Z_{c} / h$ will be set up between the two current plates, 
which will deflect the beam bunch vertically, but unfortunately in a direction opposite to the deflection by the magnetic flux density. The ratio of the electric force to the magnetic force is, using Eq. (10.2),

$$
r=\frac{\mathcal{E}}{\beta c \mathcal{B}}=\frac{2 w}{h} \frac{Z_{c}}{Z_{0}},
$$

which just equals $v_{p} / c$ and is 0.265 if $w=h$. Thus to accomplish the necessary kick the current pulse has to be increased to

$$
I=\frac{\beta E \Delta y^{\prime} w}{e Z_{0} \ell} \frac{1}{1-r} \approx 3.25 \mathrm{~A}
$$

and the power becomes $P=1.06 \mathrm{~kW}$. The peak voltage of the kicker pulse is $I Z_{c}=162.5 \mathrm{~V}$. Both the power and voltage are not high at all.

There has been an idea to lower the power consumption by lowering the characteristic impedance $Z_{c}$. This can be done, for example, by connecting 6 RG58 cables in parallel so that $Z_{c}$ is reduced 6 folds to $8.33 \Omega$. More capacitors have to be inserted between the current plates and the kicker shell to bring down the characteristic impedance. The result will reduce the phase velocity in the current plates by 6 folds to 0.0442c. The current required will also be reduced because the ratio of the electric kick to magnetic kick in the wrong direction will be lowered at the same time 6 folds to 0.0442 . The power consumption now becomes only $0.104 \mathrm{~kW}$. However, the filling time of the 1-meter damper becomes $\ell / v_{g}=75.5 \mathrm{~ns}$, which is longer than the half bunch spacing in the $140 \times 121$ scenario. Therefore, this idea is not practical.

Now let us discuss a vertical electric kicker, which consists of two horizontal voltage plates of width $w$, length $\ell$, and separated by a vertical distance $h$. The two plates are connected at the upstream ends to a voltage source via a $50-\Omega$ coaxial cable. The downstream ends are terminated by a $Z_{c}=50 \Omega$ resistor. To fire the kicker, a voltage pulse is sent into one of the horizontal plates to kick the bunch that passes by. We also require the voltage pulse to be absorbed totally by the termination resistor without reflection. Thus the two horizontal plates need to behave like a transmission line of characteristic impedance $Z_{c}$ also. The capacitance of the plates per unit length is

$$
\frac{C}{\ell}=\frac{w}{c h Z_{0}} \sim 8.85 \mathrm{pF} / \mathrm{m},
$$

when $w \sim h$. This is smaller than the capacitance per unit length

$$
\mathcal{C}=\frac{1}{c Z_{c}}=66.7 \mathrm{pF} / \mathrm{m}
$$

of a RG58 cable. On the other hand, the two voltage plates as a transmission line will support a phase velocity equal to the velocity of light. The inductance per unit length is therefore

$$
\frac{L}{\ell}=\frac{\ell}{c^{2} C}=\frac{h Z_{0}}{c w}=1.26 \mu \mathrm{H} / \mathrm{m}
$$

which is larger than the inductance per unit length of the RG58 cable. This implies a characteristic impedance of

$$
\left.Z_{c}\right|_{\text {plates }}=\frac{L}{C}=\frac{\ell}{c C}=\frac{h Z_{0}}{w},
$$

which is close to $Z_{0}=377 \Omega$ if $w \sim h$. One way to reduce the characteristic impedance to $Z_{c}=50 \Omega$ is to add capacitors between the two voltage plates. This will increase the capacitance between the two plates to

$$
\frac{C}{\ell}=\frac{L}{\ell Z_{c}^{2}} \text {, }
$$


and the phase velocity reduced to

$$
\frac{v}{c}=\frac{\ell Z_{c}}{c L}=\frac{w}{h} \frac{Z_{c}}{Z_{0}}
$$

which is $\sim 0.132$ if $w \sim h$.

Now suppose the lower plate is grounded and the a voltage pulse $V$ is passed into the upper plate, a positively-charged beam particle will be deflected downward by a force $e V / h$. This voltage pulse will have a current of $I=V / Z_{c}$, which generate a magnetic flux density $\mathcal{B}$ and will deflect the positive charge beam upward instead by the force $\sim e \beta c \mathcal{B}$. Because there is no ferrite, the magnetic field $\mathcal{H}$ will be about one half less than that given by Eq. (10.2) and so will be the flux density $\mathcal{B}$. The ratio of the electric to magnetic forces is

$$
r=\frac{\mathcal{E}}{\beta c \mathcal{B}} \sim \frac{2 w}{h} \frac{Z_{c}}{Z_{0}}
$$

which is about twice the phase velocity and is $\sim 0.265$ when $w \sim h$. Since the magnetic kick is larger than the electric kick, this is in essence a magnetic kicker without ferrite. It appears that there is no transmission-line kicker that relies mainly on electric kicks.

If we just consider the magnetic kick, the current required to produce a $\Delta y^{\prime}=1 \times 10^{-7}$ kick is

$$
I \approx \frac{2 \beta E \Delta y^{\prime} w}{e Z_{0} \ell} \frac{1}{1-r} \approx 6.50 \mathrm{~A} .
$$

This is twice the current needed in the magnetic kicker with ferrite and the reason is that without ferrite it takes about twice the current to produce the same magnetic flux density. The power consumption is then $P=I^{2} Z_{c}=2.12 \mathrm{~kW}$, which is twice the power for the ferrite kicker. This is because the current has been doubled but there is only one circuit providing the current or voltage pulse.

In reality, it is difficult to have the two plates to form a transmission line, because the plates are often close to the wall of the beam pipe or the kicker wall. For this reason, each plate should be considered separately as a transmission line with respect to the kicker shell be powered by separate sources. However, this will be exactly the same as the magnetic kicker we first discussed but in the absence of ferrite.

Usually. the particle beam is encircled in a ceramic pipe inside the kicker. If the ceramic pipe has a thin layer of metallic coating, the beam particles will be blocked from all static electric fields. Under this situation, the electric force that kicks the beam in the wrong direction will be eliminated.

The kicker system discussed above will be able to damp any transverse coupled-bunch instabilities in the dipole $(m=0)$ mode produced by wake fields between bunches that give an average transverse deflection at $150 \mathrm{GeV}$ of

$$
\frac{\Delta y^{\prime}}{\Delta y} \leq\left.\frac{\Delta y^{\prime}}{\Delta y}\right|_{\text {kicker }}=\frac{1}{\beta_{y} n}=2.0 \times 10^{-4} \mathrm{rad} / \mathrm{m} / \text { turn }
$$

The resistive-wall impedance that drives the transverse coupled-bunch instability has the value of $\left|\operatorname{Re} Z_{\perp}\right|=66.7 \mathrm{M} \Omega / \mathrm{m}$ at frequency $-0.43 f_{0}$. For 140 proton bunches. it will create in each turn a deflection of

$$
\frac{\Delta y^{\prime}}{\Delta y}=\frac{e M I_{b}\left|\operatorname{Re} Z_{\perp}\right|}{2 E}=6.4 \times 10^{-5} \mathrm{rad} / \mathrm{m} / \mathrm{turn}
$$


Therefore, the damper studied above should be able to damp this instability as well. We would like to point out that the resistive-wall driven coupled-bunch instability oscillates with a very low frequency so that there is no need to use such a fast damper considered here.

\section{CONCLUSIONS}

(1) We have estimated the longitudinal and transverse impedances of the Tevatron vacuum chamber. Below $10 \mathrm{MHz}$, the resistive wall and the Lambertson magnets dominate. Between $10 \mathrm{MHz}$ and $1 \mathrm{GHz}$, the inductive parts of the bellows and BPM contributions become important. The resonant peaks of the separators and the bellows show up above $1 \mathrm{GHz}$. Therefore, from 0.5 to a few $\mathrm{GHz}$, it is reasonable to assume that $\operatorname{Re} Z_{\|} / n \approx \operatorname{Im} Z_{\|} / n \sim 3 \Omega$ and $\operatorname{Re} Z_{\perp} \approx \operatorname{Im} Z_{\perp} \sim 3 \mathrm{M} \Omega / \mathrm{m}$.

For a rms bunch length of $37 \mathrm{~cm}$, microwave instabilities, both longitudinal and transverse, can be driven by frequencies as low as $\sim 0.1 \mathrm{GHz}$. Thus lowering the impedances in the low-frequency region is important. For example, if the laminations of the Lambertson magnets can be shielded, the impedances can be reduced by about $2 / 3$.

(2) Potential-well distortion by the inductive part of the longitudinal impedance is not severe. The bunch will be lengthened by about 1.5 to $2.0 \%$.

(3) Longitudinal microwave instability is way below the Boussard-modified Keil-Schnell limit, and therefore should not occur.

(4) Longitudinal coupled-bunch instability driven by the higher-order modes in the Tevatron cavity will be a problem. A longitudinal bunch-by-bunch damper for 7-bucket spacing is required. We also suggest passive damping of these higher-order modes.

(5) Because in Run II the bunch length will be shorter than in the Run I, the growth of longitudinal head-tail modes driven by the fundamental $\mathrm{rf}$ resonance should be much reduced. However, their growths driven by the broad band impedance will be increased.

(6) It appears that the transverse microwave instability is slightly over the stability limit of damping by momentum spread. However, operating with a positive chromaticity, for example $\xi=+10$, will be able to stabilize the situation.

(7) The transverse coupled-bunch instability driven by the resistive wall impedance appears to be the most severe collective instability here. For the $140 \times 121$ scenario, the growth rate can be as high as $75 \mathrm{~s}^{-1}$. It is lowered to $15 \mathrm{~s}^{-1}$ even when the ring is operated with the chromaticity of $\xi=+10$. However, with such a high chromaticity the $m=1$ quadrupole mode may have a sizable growth rate. Therefore, a transverse damper is necessary to cope with the situation. Although the transverse coupled-bunch instabilities can also be driven by the higher-order modes in the rf cavities, the growth rates are found to be insignificantly small.

(8) Transverse head-tail instability driven by the short-range part of the wake can occur only when 
the chromaticity is nonzero. It is driven mostly by the resistive wall impedance. If $\xi=+10$ say, the $m=0$ mode is stable while the $m=1$ mode has a growth rate of $\sim 4 \mathrm{~s}^{-1}$ and can be damped by a small tune spread. All the higher modes have much smaller growth rates.

(9) We estimate that to damp an injection error of $0.5 \mathrm{~mm}$ in 50 turns at a location where the betatron function is $\beta=100 \mathrm{~m}$, the transverse kicker must have a minimum power of $355 \mathrm{~W}$. Such a bunch-by-bunch kicker system will also be powerful enough to damp all transverse coupled-bunch instabilities in the dipole $m=0$ mode.

\section{ACKNOWLEDGMENT}

The author wishes to thank Drs. Zubao Qian and Bruno Zotter for discussions and comments, and also their reading of more than one drafts of the manuscript. 


\section{References}

[1] K.Y. Ng, Part. Accel. 16, 63 (1984).

[2] A.W. Chao, Physics of Collective Beam Instabilities in High Energy Accelerators, John Wiley \& Sons, Inc., 1993, p. 70.

[3] K.Y. Ng, Part. Accel. 23, 93 (1988).

[4] Y.H. Chin, User's Guide for New ABCI Version 6.2 (Azimuthal Beam Cavity Interaction), LBL report LBL-33091 (UC-414), 1992.

[5] MAFIA User Guide, DESY, LANL and KFA, May 3, 1988.

[6] F.A. Harfoush and K.Y. Ng, Study of Separators using Numerical Simulations, Fermilab Report FN$536,1990$.

[7] D. Sun, private communication.

[8] see for example, K.L.F. Bane, P.B. Wilson, and T. Weiland, AIP Proc. 127, Phys. of High Energy Accel., BNL/SUNY, 1983, p.875.

[9] A. Hofmann, Frontiers of Particle Beams, Lecture notes in Phys., 296, Springer-Verlag, 1986, p.99.

[10] E. Keil and W. Schnell, CERN/ISR-TH/69-48, 1969.

[11] D. Boussard. CERN/PS-BI, 1972.

[12] F.J. Sacherer, IEEE Trans. Nuclear Sci. NS 20, 3, 825 (1973).

[13] D. Boussard and T. Linnecar, Proc. 2nd European Particle Accelerator Conference, Nice, 1990, edited by P. Marin and P. Mandrillon (Editions Frontieres, Gif-sur-Yvette Cedex, France, 1990), 1560.

[14] see for example, B. Zotter, Theoretical Aspects of the Behaviour of Beams in Accelerators and Storage Rings, Proc. First Course of Int. School of Part. Accel., Erice, Nov. 10-22, 1976, p.176.

[15] F.J. Sacherer, Theoretical Aspects of the Behaviour of Beams in Accelerators and Storage Rings, Proc. First Course of Int. School of Part. Accel., Erice, Nov. 10-22, 1976, p.198.

[16] F.J. Sacherer, Methods for Computing Bunched-Beam Instabilities, CERN Report CERN/SI-BR/72-5, 1972. 